\title{
Third Semi-Annual Groundwater Monitoring Report for the Interim Remedial Action D Area Oil Seepage Basin
}

by

E. Palmer

Westinghouse Savannah River Company

Savannah River Site

Aiken, South Carolina 29808

DOE Contract No. DE-AC09-96SR18500

This paper was prepared in connection with work done under the above contract number with the U.S.

Department of Energy. By acceptance of this paper, the publisher and/or recipient acknowledges the U.S. Government's right to retain a nonexclusive, royalty-free license in and to any copyright covering this paper, along with the right to reproduce and to authorize others to reproduce all or part of the copyrighted paper.

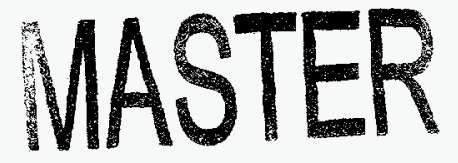

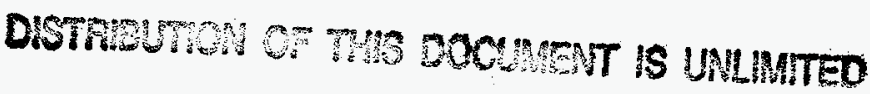

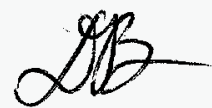




\section{DISCLAIMER}

This report was prepared as an account of work sponsored by an agency of the United States Government. Neither the United States Government nor any agency thereof, nor any of their employees, makes any warranty, express or implied, or assumes any legal liability or responsibility for the accuracy, completeness, or usefulness of any information, apparatus, product, or process disclosed, or represents that its use would not infringe privately owned rights. Reference herein to any specific commercial product, process, or service by trade name, trademark, manufacturer, or otherwise does not necessarily constitute or imply its endorsement, recommendation, or favoring by the United States Government or any agency thereof. The views and opinions of authors expressed herein do not necessarily state or reflect those of the United States Government or any agency thereof.

This report has been reproduced directly from the best available copy.

Available to DOE and DOE contractors from the Office of Scientific and Technical Information, P.O. Box 62, Oak Ridge, TN 37831; prices available from (615) 576-8401.

Available to the public from the National Technical Information Service, U.S. Department of Commerce, 5285 Port Royal Road, Springfield, VA 22161. 


\section{DISCLAIMER}

Portions of this document may be illegible electronic image products. Images are produced from the best available original document. 


\title{
THIRD SEMI-ANNUAL GROUNDWATER MONITORING REPORT FOR THE D-AREA OIL SEEPAGE BASIN INTERIM REMEDIAL ACTION
}

\author{
WSRC-RP-98-4110
}

JUNE 18, 1998

Written by: Greg Rucker

Site Geotechnical Services

Projects, Engineering and Construction Division

Westinghouse Savannah River Company

Savannah River Site

Aiken, SC 29808

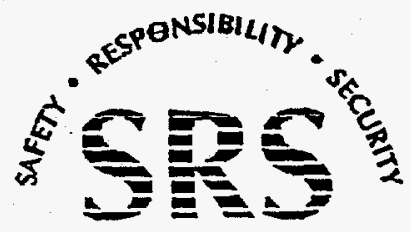




\section{Table of Contents}

1.0 PURPOSE 1

2.0 HISTORY 1

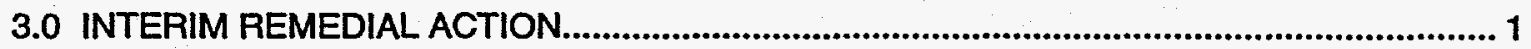

4.0 REGULATORY BASIS FOR GROUNDWATER MONITORING .....................................2

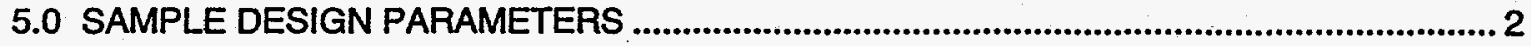

6.0 BACKGROUND GROUNDWATER CONCENTRATIONS .............................................. 6

7.0 COMPARISON OF POST-IRA SAMPLES TO BACKGROUND VALUES ......................... 12

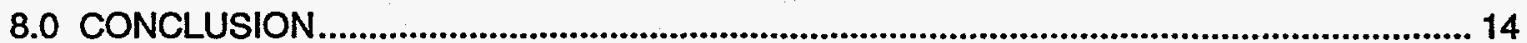

\section{List of Tables}

Table 5-1: Monitoring Well Screen Zones in Feet Above Mean Sea LeVel (MSL) .................. 3

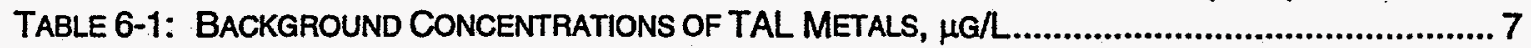

TABLE 6-2: BACKGROUND CONCENTRATIONS FOR TCL VOLATILE ORGANIC COMPOUNDS, $\mu$ G/L.......... 9

TABLE 6-3: BACKGROUND CONCENTRATIONS FOR INDICATOR PARAMETERS, $\mu \mathrm{G} / \mathrm{L} \ldots \ldots \ldots \ldots \ldots \ldots . . . . . . . . .11$

TABLE 7-1: MAXIMUM CONCENTRATIONS DETECTED IN POST-IRA GROUNDWATER SAMPLES, $\mu \mathrm{G} / L . . .15$

TABLE 7-2: COMPARISON OF POST-IRA SAMPLES TO PREDICTED TOLERANCE INTERVALS, $\mu$ G/L ....... 16

TABLE 7-3: COMPARISON OF MAXIMUM CONCENTRATION DETECTED IN PRE-IRA SAMPLES TO MAXIMUM

CONCENTRATION IN POST-IRA SAMPLES, $\mu \mathrm{G} / L$

\section{Figures}

Figure 5-1: D-Area Oil Seepage Basin Showing Location of Monitoring Wells and Piezometers. 


\subsection{PURPOSE}

The purpose of this report is to evaluate groundwater quality data collected from six downgradient groundwater monitoring wells for potential impact caused by the Interim Remedial Action (IRA) (completed June 1996) at the D-Area Oil Seepage Basin. The US Environmental Protection Agency and South Carolina Department of Health and Environmental Control required post-IRA groundwater monitoring and this report.

\subsection{HISTORY}

D-Area Oil Seepage Basin (DOSB), Bldg. 631-G is located north of D-Area between Roads A-4.4 and A-4.5 at an elevation approximately 150 feet above mean sea level and 1.9 miles from the nearest SRS boundary (Savannah River). The major surface water drainage system is the Savannah River and associated swamps located approximately 1.3 miles west of the basin. Upper Three Runs Creek is located 1.7 miles to the north-northwest; Fourmile. Branch is 1.7 miles to the south-southeast.

A detailed history for the DOSB can be found in the Resource Conservation and Recovery Act (RCRA) Facility Investigation/Remedial Investigation and the Baseline Risk Assessment, WSRC-RP-96-154, Rev. 1. The DOSB was originally constructed in 1952 as a series of unlined trenches to dispose of waste oils and other fluids not suitable for burning in powerhouse boilers. These waste oils included seal oil from the Heavy Water Facility, machine cutting oil, transformer oil and other shop fluids. The waste oils and fluids were collected in 55-gallon drums, transported to the basin, opened and dumped into the trenches. As the trenches filled, they were alternately burned along with general office and cafeteria waste. This practice continued until 1973 when open burning ceased plant wide.

In 1975, the trenches were removed from service and backfilled with soil (WSRC, 1990). Approximately one foot of standing water, plus a number of 55-gallon drums, possibly containing waste oil, remained in the basin as documented in a DOSB photograph (\#17-355-40) taken in the 1970s.

\subsection{INTERIM REMEDIAL ACTION}

An IRA was planned for the first quarter of 1995 to remove the drums, debris, and principal threat source material such as free product and sludge. The IRA was planned for completion before initiation of the remedial investigation. However, due to a higher than expected water table, the IRA was postponed until a plan could be devised to minimize impact from excavation within the shallow water table. Approval of the Remedial Design/Remedial Action work plan (RD/RA) (WSRC-RP-94-1287, Rev. 1.3) was granted in March 1996 and the IRA was completed in June of 1996. The IRA was conducted to reduce the immediate health and environmental contact potential with the contaminant source by uncovering the drums and debris through careful excavation, transferring the drum contents into new drums, and dispositioning of the drum contents and debris in accordance with the approved RDRA.

Metal objects removed during the IRA included partially crushed drums with product and sludge, empty crushed drums, wire, chain, cable, conduit metal shavings, metal strips, glass bottles, light bulbs, reagent bottles, glassware, plastic sheeting, canvas, cloth, bricks, large ( 8 inch and 4 inch) pipes, rip-rap, channel iron, rebar, etc. Some drums were buried for at least 17 years and were still intact when excavated during the IRA while others were not. Few of the, approximately 60 , excavated containers contained free liquids and/or residual sludges (less than 110 gallons). 
The excavation method utilized during the IRA was conducted by opening small quadrants (10 $\mathrm{x}$ 20 feet) of the waste unit to lessen the potential for environmental impacts due to inadvertent releases (e.g., leakage from drum contents into the shallow water table) of principal threat source material. Soil removed during the excavation process was placed back in the quadrants in a last-out-first-in basis to ensure that the cleanest soil was placed nearest the surface. After all the quadrants were excavated, clean fill was placed on top of the basin area until the soil was approximately one foot above the existing grade. The soils were then graded to reduce water infiltration and seeded to prevent erosion.

Extensive characterization activities have also been completed at the DOSB including four phases of solls and groundwater investigation utilizing the Streamlined Approach for Environmental Restoration (SAFER) and Expedited Site Characterization (ESC) methodology. These characterization activities are documented in detail in the RCRA Facility Investigation/Remedial Investigation and Baseline Risk Assessment Report WSRC-RP-96-154, Rev. 1.

\subsection{REGULATORY BASIS FOR GROUNDWATER MONITORING}

The regulatory requirements for this groundwater report originate in the RD/RA Work Plan Section 4.2.9. Before the IRA began, concern was expressed by the regulators that the proposed activities during the IRA could potentially degrade DOSB groundwater quality. Specifically, it was known that the water table elevation was above the bottom of the basin trenches and that excavation and drum handling would have to occur within the water table. The proposed excavation and drum removal could potentially cause the drum contents to spill or leak into the groundwater, worsening the existing groundwater quality.

SCDHEC and USEPA mandated that a detailed groundwater monitoring strategy be included in the RD/RA work plan and implemented prior to and following the completion of any excavation at the site to ensure early detection in the event of a release from the IRA.

As a result, a number of operational modifications were made to the excavation and drum handling procedures in the IRA to minimize any leakage of drum contents into the aquifer including a requirement for semi-annual groundwater monitoring after the IRA was completed.

Under the terms of the approved RD/RA work plan sampling and analysis plan:

1. Existing monitoring wells in proximity to the IRA were to be selected for detection monitoring.

2. Since it was known that downgradient groundwater had already been impacted, comparison of background data to downgradient data would not indicate releases from the IRA.

3. Changes in groundwater quality would be indicated by exceeding predicted tolerance intervals and the magnitude of those changes would be assessed and reported to USEPA and SCDHEC.

4. Groundwater would be sampled semi-annually for three years.

5. A report of the groundwater sampling would be presented to USEPA and SCDHEC, semiannually, summarizing the results of the water quality analysis.

\subsection{SAMPLE DESIGN PARAMETERS}

Groundwater monitoring wells from the existing monitoring well and piezometer network surrounding DOSB were utilized for detection monitoring before, during and after the IRA. 
Groundwater monitoring wells DOB-1, DOB-2, DOB-7, DOB-8, DOB-10 and DOB-12 were selected for monitoring because they are the closest to the excavated area. These wells will be the first to be impacted if a release of hazardous substances occurred during the IRA. These wells are located at the waste. unit perimeter directly downgradient and side-gradient of the DOSB and will provide the earliest possible detection of any releases from the IRA. See Figure 5-1.

Table 5-1: Monitoring Well Screen Zones in Feet Above Mean Sea Level (MSL)

\begin{tabular}{|c|c|c|c|c|c|}
\hline Well & $\begin{array}{c}\text { Screen Top } \\
(\text { MSL) }\end{array}$ & $\begin{array}{c}\text { Screen Bottom } \\
(\text { MSL) }\end{array}$ & $\begin{array}{c}\text { Ground Surface } \\
(M S L)\end{array}$ & $\begin{array}{c}\text { Screen Length } \\
(\mathrm{ft})\end{array}$ & $\begin{array}{c}\text { Screen Depth(ft) } \\
\text { (Below Surface) }\end{array}$ \\
\hline DOB-1 & 144.7 & 114.7 & 149.7 & 30 & 5 \\
\hline DOB-2 & 145.3 & 115.3 & 150.3 & 30 & 5 \\
\hline DOB-7 & 145.7 & 125.7 & 148.7 & 20 & 3 \\
\hline DOB-8 & 148.3 & 128.3 & 151.3 & 20 & 3 \\
\hline DOB-10 & 148.3 & 128.3 & 153.5 & 20 & 5.2 \\
\hline DOB-12 & 137.9 & 132.9 & 149.6 & 5 & 11.7 \\
\hline
\end{tabular}

Because groundwater downgradient of the DOSB is known to be impacted from contaminants previously released from DOSB, comparison of background data to downgradient data would not indicate a release from the IRA. The sampling design for groundwater monitoring is based upon the establishment of background conditions in the above designated down and side-gradient wells. In effect, data collected from each well, prior to and during the IRA, will serve to establish its own background quality.

Prior to the beginning and during the IRA, 10 background samples were collected and analyzed from each well from April 4, 1996, to June 5, 1996. The results for each constituent and each well were used to calculate a predicted tolerance level (upper confidence level). The tolerance interval was calculated at the $n-1,0.05(95 \%)$ level of significance and was one-tailed $(+)$, except for $\mathrm{pH}$ which was two-tailed $(+/-)$. The level of significance is consistent with RCRA requirements (RCRA 264.1066, Appendix IV).

The tolerance interval was computed by:

$$
P T I=X_{\text {bar }} \pm t * \frac{S D}{\sqrt{n}}
$$

Where:

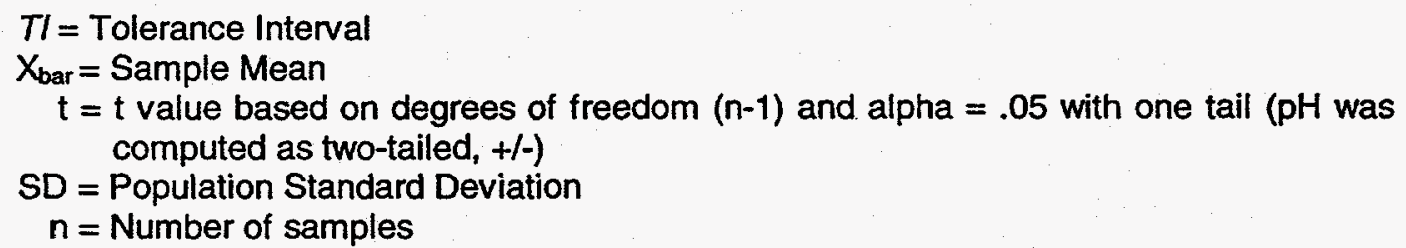




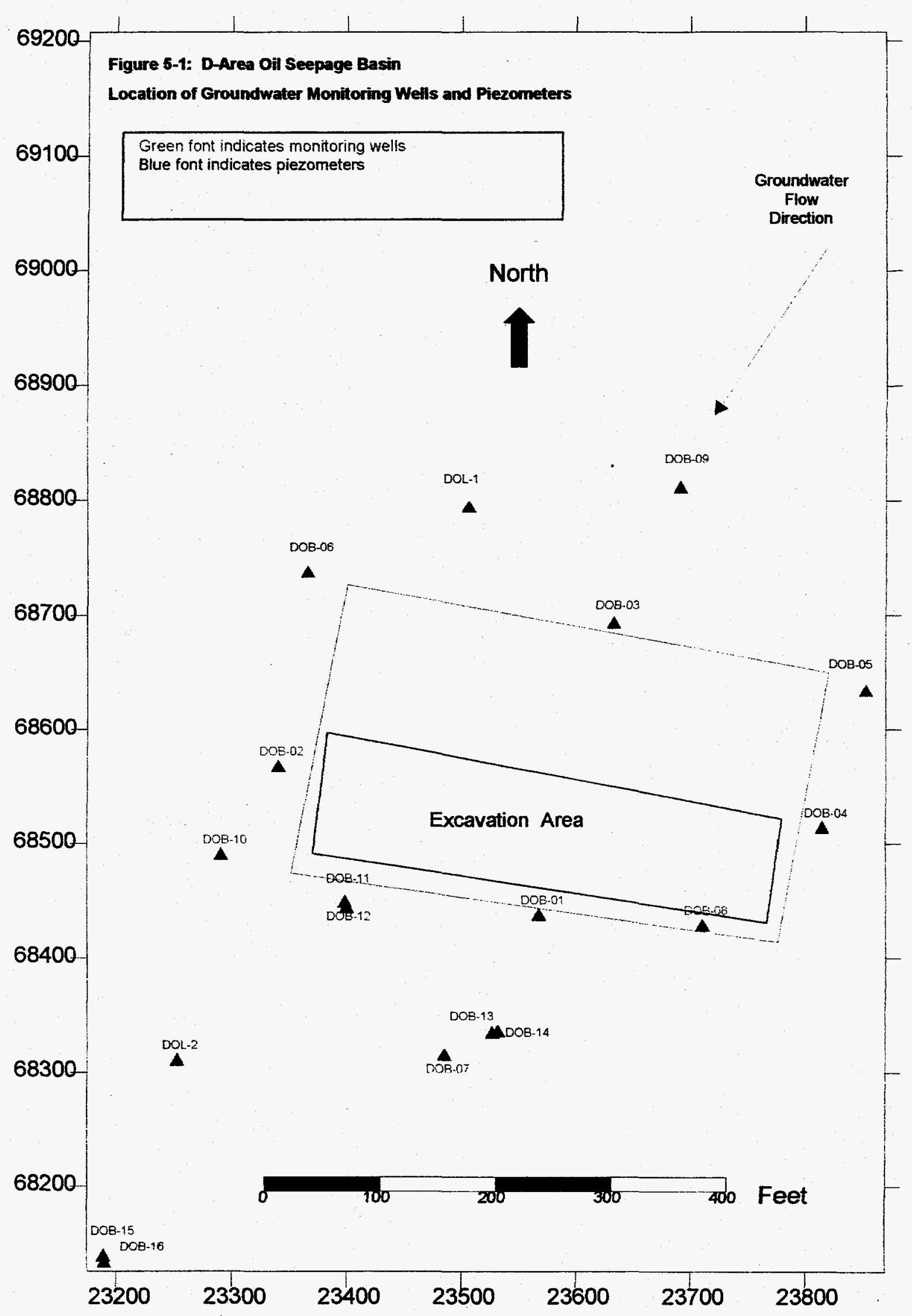


After background conditions have been calculated for each well, groundwater samples are to be collected on a semi-annual basis for a three year period of time following completion of the IRA and compared to the tolerance interval. Exceedance of the tolerance interval could indicate a change in groundwater quality; however, it may also reflect change due to statistical aberrations or be attributable to temporal variability or sample collection and measurement error. Therefore, the exceedance of a tolerance interval would not necessarily trigger further groundwater investigation. Exceedance of a tolerance interval would necessitate an evaluation of the body of data to determine if the impact to groundwater quality was positive or negative.

A three year monitoring duration was estimated based upon the distance from the center (75 feet) and far edge (150 feet) of the IRA excavation area and the range of groundwater flow velocities found at DOSB. The following was used to estimate the three-year sampling duration.

$$
\begin{aligned}
& V \approx \frac{K \cdot\left(\frac{\Delta h}{\Delta l}\right)}{n_{e}} \\
& V \approx \frac{9.7 \text { to } 32.0 \mathrm{ft} / \text { day } \cdot(.003)}{.24} \approx 0.12 \text { to0.40 ft / day } \\
& D \approx 0.12 \text { to } 0.40 \mathrm{ft} / \text { day } \cdot(365 \text { days / year) } \approx 44 \text { tol } 46 \mathrm{feet} / \mathrm{year} \\
& T_{1}=75 \text { feet (midpoint of excavated area to well)/44 to } 146 \mathrm{ft} / \mathrm{yr} \\
& I_{1}=0.51 \text { to } 1.7 \text { years travel time (high range estimate) } \\
& T_{2}=150 \text { feet (far edge of excavated area to well)/44 to } 146 \mathrm{ft} / \mathrm{yr} \\
& T_{2}=1.02 \text { to } 3.4 \text { years travel time (low range estimate) } \\
& \text { Assuming: } \\
& V=\text { Average linear velocity feet per day } \\
& K=\text { Hydraulic conductivity range } 18.8 \text { to } 32.0 \text { feet/day } \\
& h=\text { height feet } \\
& I=\text { length feet } \\
& n_{\theta}=\text { effective porosity .24\% } \\
& D=\text { distance plume has migrated in feet per year } \\
& T_{1}=\text { Travel Time range estimate from midpoint in years } \\
& T_{2}=\text { Travel Time range estimate from far edge in years }
\end{aligned}
$$

The monitoring data results from each semi-annual sampling event will be compared to the tolerance intervals. If the tolerance interval is exceeded, then the magnitude of the change will be assessed with respect to the overall body of data and trending. The results will be reported to the South Carolina Department of Health and Environmental Control and the US Environmental Protection Agency twice each year.

The constituents selected for monitoring include Target Compound List Volatile Organic Compounds (TCL-VOCs), Target Analyte List Metals (TAL) and indicator parameters pH, specific conductance, Chemical Oxygen Demand (COD), nitrates, sulfates, chlorides, Total Organic Carbon (TOC), and Total Organic Halogens (TOX). These analytes were selected for monitoring 
because they represent the waste sources characteristic of DOSB and because they are the most mobile, with the exception of TAL metals. Therefore, the analyte list has been specifically tuned to be representative and responsive to detection during compliance monitoring.

\subsection{BACKGROUND GROUNDWATER CONCENTRATIONS}

Ten background groundwater samples were collected from each of six downgradient wells from April 4, 1996, to June 5, 1996. Ten samples were selected for collection to reduce statistical error and reasonably limit the upper-bound. The monitoring wells sampled included DOB-1, DOB-2, DOB-7, DOB-8, DOB-10 and DOB-12 and are the wells closest to the waste unit.

All samples were analyzed in accordance with SW-846 methods. All dafa used in this evaluation is definitive level validated data. Because groundwater downgradient of DOSB is impacted from previous releases, a method to measure change in water quality in the wells post-IRA is by comparing post-IRA sample results to a $95 \%$ tolerance interval.

Excluding quality assurance/quality control (QAVC) samples, the background data set consisted of 3973 lines of analytical data. The data set was purged to eliminate QAVQC samples, replicates, and rejected data. Background groundwater statistics including the average and $95 \%$ tolerance levels were calculated for the analyte concentrations in each well. Where a sample result included a " $U$ " qualifier, indicating analyte concentrations were below sample quantitation limits in one or more background samples, a surrogate value of one-half the sample quantitation limit was used to calculate the average for that analyte. Where a particular analyte was not detected in any of the background samples, the background concentration for comparison purposes was set to zero, to guarantee that any detection in post-IRA samples would be addressed. Simple summary statistics were then generated for each analytical fraction by well. The results of the background summary statistics are presented on Tables 6-1, 6-2 and 6-3.

Background groundwater samples were analyzed for 20 metals, as shown on Table 6-1. In background samples, 19 of the 20 analytes (excepting only silver) were detected.

Background groundwater samples were analyzed for 34 TCL VOCs, as shown on Table 6-2. In the background samples, 17 of the 34 analytes (excepting 1,1,1-trichloroethane, 1,1,2,2,tetrachloroethane, 1,1,2-trichloroethane, 1,1,-dichloroethane, 1,2-dichloropropane, 2-hexanone, bromodichloromethane, bromoform, bromomethane, chloroethane, chloromethane, cis-1,3dichloropropene, dibromochloromethane, methyl isobutyl ketone, styrene, trans-1,3dichloropropene, and vinyl acetate) were detected.

Background groundwater samples were analyzed for 8 indicator parameters as shown on Table 6-3. All indicator parameters were measured in the background samples. 
Table 6-1: Background Concentrations of TAL Metals, $\mu \mathrm{g} / \mathrm{L}$

\begin{tabular}{|c|c|c|c|c|c|c|c|c|}
\hline & well_name & DOB 1 & DOB 2 & DOB 7 & DOB 8 & DOB 10 & DOB 12 & Summary \\
\hline Analyte & Data. & & & & & & & \\
\hline Aluminum & $\begin{array}{l}\text { Total Samples } \\
\text { Hits } \\
\text { Minimum Detection } \\
\text { Maximum Detection } \\
\text { Mean }\end{array}$ & \begin{tabular}{r|}
10 \\
7 \\
47.5 \\
774 \\
213.06
\end{tabular} & \begin{tabular}{r|}
11 \\
6 \\
16 \\
107 \\
42.09 \\
\end{tabular} & $\begin{array}{r}10 \\
7 \\
77 \\
138 \\
90.28\end{array}$ & \begin{tabular}{r|}
10 \\
7 \\
30.1 \\
180 \\
73.32 \\
\end{tabular} & $\begin{array}{r}10 \\
8 \\
12.6 \\
2170 \\
284.97\end{array}$ & \begin{tabular}{r|}
10 \\
7 \\
16.6 \\
179 \\
53.59
\end{tabular} & \begin{tabular}{r|}
61 \\
42 \\
12.6 \\
2170 \\
124.84 \\
\end{tabular} \\
\hline Antimony & $\begin{array}{l}\text { Total Samples } \\
\text { Hits } \\
\text { Minimum Detection } \\
\text { Maximum Detection } \\
\text { Mean } \\
\end{array}$ & \begin{tabular}{r|}
10 \\
2 \\
1.38 \\
1.64 \\
2.30 \\
\end{tabular} & \begin{tabular}{r|}
10 \\
0 \\
0 \\
0 \\
2.50
\end{tabular} & \begin{tabular}{r|r|}
10 \\
0 \\
0 \\
0 \\
2.50 \\
\end{tabular} & $\begin{array}{r}10 \\
0 \\
0 \\
0 \\
2.50 \\
\end{array}$ & $\begin{array}{r}10 \\
0 \\
0 \\
0 \\
2.50 \\
\end{array}$ & $\begin{array}{r}10 \\
0 \\
0 \\
0 \\
2.50 \\
\end{array}$ & \begin{tabular}{r|}
60 \\
2 \\
1.38 \\
1.64 \\
2.47 \\
\end{tabular} \\
\hline Arsenic & $\begin{array}{l}\text { Total Samples } \\
\text { Hits } \\
\text { Minimum Detection } \\
\text { Maximum Detection } \\
\text { Mean } \\
\end{array}$ & $\begin{array}{r}10 \\
1 \\
2.11 \\
2.11 \\
2.46 \\
\end{array}$ & $\begin{array}{r}10 \\
1 \\
2.03 \\
2.03 \\
2.45\end{array}$ & $\begin{array}{r}10 \\
0 \\
0 \\
0 \\
2.50\end{array}$ & \begin{tabular}{r|}
10 \\
0 \\
0 \\
0 \\
2.50 \\
\end{tabular} & \begin{tabular}{r|}
10 \\
1 \\
1.99 \\
1.99 \\
2.45 \\
\end{tabular} & \begin{tabular}{r|}
10 \\
0 \\
0 \\
0 \\
2.50
\end{tabular} & $\begin{array}{r}60 \\
3 \\
0 \\
2.11 \\
2.48 \\
\end{array}$ \\
\hline Barium & $\begin{array}{l}\text { Total Samples } \\
\text { Hits } \\
\text { Minimum Detection } \\
\text { Maximum Detection } \\
\text { Mean }\end{array}$ & $\begin{array}{r}10 \\
10 \\
10.9 \\
16.2 \\
14.10 \\
\end{array}$ & $\begin{array}{r}10 \\
10 \\
26.2 \\
31 \\
28.34 \\
\end{array}$ & $\begin{array}{r}10 \\
10 \\
43.5 \\
52.2 \\
47.19 \\
\end{array}$ & $\begin{array}{r}10 \\
10 \\
14 \\
23.2 \\
18.40 \\
\end{array}$ & $\begin{array}{r}10 \\
10 \\
15.1 \\
33.5 \\
19.40 \\
\end{array}$ & $\begin{array}{r}10 \\
10 \\
16.8 \\
20.7 \\
19.10 \\
\end{array}$ & $\begin{array}{r}60 \\
60 \\
10.9 \\
52.2 \\
24.42 \\
\end{array}$ \\
\hline Beryllium & $\begin{array}{l}\text { Total Samples } \\
\text { Hits } \\
\text { Minimum Detection } \\
\text { Maximum Detection } \\
\text { Mean }\end{array}$ & \begin{tabular}{r|}
10 \\
0 \\
0 \\
0 \\
0.61
\end{tabular} & $\begin{array}{r}10 \\
2 \\
0.017 \\
0.026 \\
0.33\end{array}$ & $\begin{array}{r}10 \\
0 \\
0 \\
0 \\
0.62\end{array}$ & $\begin{array}{r}10 \\
1 \\
0.015 \\
0.015 \\
0.47\end{array}$ & $\begin{array}{r}10 \\
0 \\
0 \\
0 \\
0.76\end{array}$ & $\begin{array}{r}10 \\
4 \\
0.108 \\
0.211 \\
0.40 \\
\end{array}$ & $\begin{array}{r}60 \\
7 \\
0 \\
0.211 \\
0.54\end{array}$ \\
\hline Cadmium & $\begin{array}{l}\text { Total Samples } \\
\text { Hits } \\
\text { Minimum Detection } \\
\text { Maximum Detection } \\
\text { Mean } \\
\end{array}$ & $\begin{array}{r}10 \\
1 \\
0.099 \\
0.099 \\
0.91 \\
\end{array}$ & $\begin{array}{r}10 \\
1 \\
0.109 \\
0.109 \\
0.91 \\
\end{array}$ & $\begin{array}{r}10 \\
6 \\
0.107 \\
0.23 \\
0.42 \\
\end{array}$ & $\begin{array}{r}10 \\
1 \\
0.319 \\
0.319 \\
0.93 \\
\end{array}$ & $\begin{array}{r}10 \\
0 \\
0 \\
0 \\
1.00 \\
\end{array}$ & $\begin{array}{r}10 \\
0 \\
0 \\
0 \\
1.00 \\
\end{array}$ & $\begin{array}{r}60 \\
9 \\
0 \\
0.319 \\
0.86 \\
\end{array}$ \\
\hline Chromium & $\begin{array}{l}\text { Total Samples } \\
\text { Hits } \\
\text { Minimum Detection } \\
\text { Maximum Detection } \\
\text { Mean } \\
\end{array}$ & $\begin{array}{r}10 \\
5 \\
0.974 \\
2.53 \\
1.74 \\
\end{array}$ & $\begin{array}{r}10 \\
4 \\
0.851 \\
2.14 \\
1.73 \\
\end{array}$ & \begin{tabular}{r|}
10 \\
4 \\
0.714 \\
2.63 \\
1.64 \\
\end{tabular} & $\begin{array}{r}10 \\
5 \\
0.651 \\
1.98 \\
1.52 \\
\end{array}$ & \begin{tabular}{r|}
10 \\
6 \\
0.709 \\
7.73 \\
2.01 \\
\end{tabular} & \begin{tabular}{r|}
10 \\
8 \\
0.782 \\
2.67 \\
1.57 \\
\end{tabular} & $\begin{array}{r}60 \\
32 \\
0.651 \\
7.73 \\
1.70 \\
\end{array}$ \\
\hline Cobalt & $\begin{array}{l}\text { Total Samples } \\
\text { Hits } \\
\text { Minimum Detection } \\
\text { Maximum Detection } \\
\text { Mean } \\
\end{array}$ & \begin{tabular}{r|}
10 \\
3 \\
0.196 \\
0.239 \\
1.16 \\
\end{tabular} & $\begin{array}{r}10 \\
5 \\
0.286 \\
0.672 \\
0.72 \\
\end{array}$ & \begin{tabular}{r|}
10 \\
6 \\
0.284 \\
0.558 \\
0.75 \\
\end{tabular} & $\begin{array}{r}10 \\
5 \\
0.208 \\
0.523 \\
0.65 \\
\end{array}$ & $\begin{array}{r}10 \\
4 \\
0.181 \\
0.324 \\
0.78 \\
\end{array}$ & \begin{tabular}{r|}
10 \\
6 \\
0.323 \\
0.932 \\
0.79 \\
\end{tabular} & $\begin{array}{r}60 \\
29 \\
0.181 \\
0.932 \\
0.81 \\
\end{array}$ \\
\hline Copper & $\begin{array}{l}\text { Total Samples } \\
\text { Hits } \\
\text { Minimum Detection } \\
\text { Maximum Detection } \\
\text { Mean } \\
\end{array}$ & $\begin{array}{r}10 \\
10 \\
37.8 \\
96.2 \\
52.09 \\
\end{array}$ & $\begin{array}{r}10 \\
7 \\
0.592 \\
2.74 \\
1.98 \\
\end{array}$ & \begin{tabular}{r|}
10 \\
5 \\
0.616 \\
2.27 \\
1.40 \\
\end{tabular} & \begin{tabular}{r|}
10 \\
5 \\
0.558 \\
3.33 \\
1.54 \\
\end{tabular} & $\begin{array}{r}10 \\
5 \\
1.24 \\
17.4 \\
4.41 \\
\end{array}$ & \begin{tabular}{r|}
10 \\
5 \\
1.84 \\
12.3 \\
3.32 \\
\end{tabular} & $\begin{array}{r}60 \\
37 \\
0.558 \\
96.2 \\
10.79 \\
\end{array}$ \\
\hline Iron & $\begin{array}{l}\text { Total Samples } \\
\text { Hits } \\
\text { Minimum Detection } \\
\text { Maximum Detection } \\
\text { Mean } \\
\end{array}$ & $\begin{array}{r}10 \\
9 \\
30.6 \\
955 \\
220.94 \\
\end{array}$ & \begin{tabular}{r|}
11 \\
11 \\
55.5 \\
542 \\
163.46 \\
\end{tabular} & \begin{tabular}{r|}
10 \\
7 \\
10.1 \\
39.1 \\
15.70 \\
\end{tabular} & $\begin{array}{r}10 \\
8 \\
10.8 \\
110 \\
33.09 \\
\end{array}$ & \begin{tabular}{r|}
10 \\
8 \\
12.6 \\
654 \\
86.98 \\
\end{tabular} & \begin{tabular}{r|}
10 \\
9 \\
14.2 \\
130 \\
52.52 \\
\end{tabular} & $\begin{array}{r}61 \\
52 \\
10.1 \\
955 \\
96.56 \\
\end{array}$ \\
\hline Lead & $\begin{array}{l}\text { Total Samples } \\
\text { Hits } \\
\text { Minimum Detection } \\
\text { Maximum Detection } \\
\text { Mean } \\
\end{array}$ & $\begin{array}{r}10 \\
6 \\
1.37 \\
5.06 \\
2.62 \\
\end{array}$ & \begin{tabular}{r|}
10 \\
2 \\
2.1 \\
2.26 \\
2.28 \\
\end{tabular} & \begin{tabular}{r|}
10 \\
2 \\
3.47 \\
3.77 \\
2.55 \\
\end{tabular} & $\begin{array}{r}10 \\
0 \\
0 \\
0 \\
2.32\end{array}$ & $\begin{array}{r}10 \\
4 \\
1.35 \\
16.3 \\
4.67 \\
\end{array}$ & $\begin{array}{r}10 \\
0 \\
0 \\
0 \\
2.50 \\
\end{array}$ & $\begin{array}{r}60 \\
14 \\
1.35 \\
16.3 \\
2.82 \\
\end{array}$ \\
\hline Magnesium & $\begin{array}{l}\text { Total Samples } \\
\text { Hits } \\
\text { Minimum Detection } \\
\text { Maximum Detection } \\
\text { Mean } \\
\end{array}$ & $\begin{array}{r}10 \\
10 \\
7850 \\
11400 \\
8,785.00 \\
\end{array}$ & $\begin{array}{r}10 \\
10 \\
1750 \\
2070 \\
1,931.00 \\
\end{array}$ & $\begin{array}{r}10 \\
10 \\
3890 \\
4520 \\
4,163.00 \\
\end{array}$ & \begin{tabular}{r|}
10 \\
10 \\
694 \\
905 \\
775.10 \\
\end{tabular} & $\begin{array}{r}10 \\
10 \\
1800 \\
2350 \\
2,001.00 \\
\end{array}$ & $\begin{array}{r}10 \\
10 \\
765 \\
929 \\
875.20 \\
\end{array}$ & $\begin{array}{r}60 \\
60 \\
694 \\
11400 \\
3,088.38 \\
\end{array}$ \\
\hline
\end{tabular}


Table 6-1: Background Concentrations of TAL Metals, $\mu \mathrm{g} / \mathrm{L}$ (continued)

\begin{tabular}{|c|c|c|c|c|c|c|c|c|}
\hline & well name & DOB 1 & DOB 2 & DOB 7 & DOB 8 & DOB 10 & DOB 12 & Summary \\
\hline Analyte & Data & & & & & & & \\
\hline Manganese & $\begin{array}{l}\text { Total Samples } \\
\text { Hits } \\
\text { Minimum Detection } \\
\text { Maximum Detection } \\
\text { Mean }\end{array}$ & $\begin{array}{r}10 \\
10 \\
2.37 \\
8.92 \\
4.74 \\
\end{array}$ & \begin{tabular}{r|}
11 \\
11 \\
12.1 \\
17.6 \\
13.94 \\
\end{tabular} & $\begin{array}{r}10 \\
10 \\
146 \\
177 \\
158.70 \\
\end{array}$ & $\begin{array}{r}10 \\
10 \\
1.55 \\
9.68 \\
4.24 \\
\end{array}$ & $\begin{array}{r}10 \\
10 \\
6.93 \\
14.3 \\
9.85 \\
\end{array}$ & $\begin{array}{r}10 \\
10 \\
58.8 \\
70.1 \\
64.06 \\
\end{array}$ & $\begin{array}{r}61 \\
61 \\
1.55 \\
177 \\
42.12 \\
\end{array}$ \\
\hline Mercury & $\begin{array}{l}\text { Total Samples } \\
\text { Hits } \\
\text { Minimum Detection } \\
\text { Maximum Detection } \\
\text { Mean } \\
\end{array}$ & $\begin{array}{r}10 \\
1 \\
0.049 \\
0.049 \\
0.06\end{array}$ & \begin{tabular}{r|}
10 \\
1 \\
0.436 \\
0.436 \\
0.10
\end{tabular} & $\begin{array}{r}10 \\
2 \\
0.042 \\
0.224 \\
0.10\end{array}$ & $\begin{array}{r}10 \\
1 \\
0.373 \\
0.373 \\
0.10 \\
\end{array}$ & $\begin{array}{r}10 \\
2 \\
0.015 \\
0.215 \\
0.09\end{array}$ & $\begin{array}{r}10 \\
4 \\
0.025 \\
0.112 \\
0.07\end{array}$ & $\begin{array}{r}60 \\
11 \\
0 \\
0.436 \\
0.08\end{array}$ \\
\hline Nickel & $\begin{array}{l}\text { Total Samples } \\
\text { Hits } \\
\text { Minimum Detection } \\
\text { Maximum Detection } \\
\text { Mean }\end{array}$ & $\begin{array}{r}10 \\
3 \\
0.89 \\
1.93 \\
3.89 \\
\end{array}$ & \begin{tabular}{r|}
10 \\
5 \\
0.884 \\
2.51 \\
3.44
\end{tabular} & \begin{tabular}{r|}
10 \\
3 \\
0.808 \\
1.2 \\
3.80 \\
\end{tabular} & $\begin{array}{r}10 \\
1 \\
1.17 \\
1.17 \\
4.62 \\
\end{array}$ & \begin{tabular}{r|}
10 \\
2 \\
1.23 \\
5.14 \\
4.64
\end{tabular} & $\begin{array}{r}10 \\
8 \\
0.867 \\
2.43 \\
2.24 \\
\end{array}$ & $\begin{array}{r}60 \\
22 \\
\times \quad 0 \\
5.14 \\
3.77 \\
\end{array}$ \\
\hline Selenium & $\begin{array}{l}\text { Total Samples } \\
\text { Hits } \\
\text { Minimum Delection } \\
\text { Maximum Detection } \\
\text { Mean } \\
\end{array}$ & $\begin{array}{r}10 \\
0 \\
0 \\
0 \\
2.33 \\
\end{array}$ & $\begin{array}{r}10 \\
0 \\
0 \\
0 \\
2.50 \\
\end{array}$ & $\begin{array}{r}10 \\
0 \\
0 \\
0 \\
2.41\end{array}$ & $\begin{array}{r}10 \\
1 \\
1.45 \\
1.45 \\
2.23 \\
\end{array}$ & $\begin{array}{r}10 \\
0 \\
0 \\
0 \\
2.39 \\
\end{array}$ & $\begin{array}{r}10 \\
0 \\
0 \\
0 \\
2.41\end{array}$ & $\begin{array}{r}60 \\
1 \\
0 \\
1.45 \\
2.38 \\
\end{array}$ \\
\hline Thallium & $\begin{array}{l}\text { Total Samples } \\
\text { Hits } \\
\text { Minimum Detection } \\
\text { Maximum Detection } \\
\text { Mean } \\
\end{array}$ & $\begin{array}{r}10 \\
0 \\
0 \\
0 \\
2.50 \\
\end{array}$ & $\begin{array}{r}10 \\
0 \\
0 \\
0 \\
2.50 \\
\end{array}$ & $\begin{array}{r}10 \\
0 \\
0 \\
0 \\
2.50 \\
\end{array}$ & $\begin{array}{r}10 \\
1 \\
2.48 \\
2.48 \\
2.50 \\
\end{array}$ & $\begin{array}{r}10 \\
0 \\
0 \\
0 \\
2.50 \\
\end{array}$ & $\begin{array}{r}10 \\
0 \\
0 \\
0 \\
2.50 \\
\end{array}$ & $\begin{array}{r}60 \\
1 \\
0 \\
2.48 \\
2.50 \\
\end{array}$ \\
\hline Vanadium & $\begin{array}{l}\text { Total Samples } \\
\text { Hits } \\
\text { Minimum Detection } \\
\text { Maximum Detection } \\
\text { Mean } \\
\end{array}$ & $\begin{array}{r}10 \\
9 \\
0.856 \\
4.16 \\
2.26 \\
\end{array}$ & $\begin{array}{r}10 \\
5 \\
0.28 \\
0.816 \\
1.81 \\
\end{array}$ & \begin{tabular}{r|}
10 \\
3 \\
0.266 \\
0.387 \\
3.11 \\
\end{tabular} & $\begin{array}{r}10 \\
5 \\
0.316 \\
0.818 \\
0.85 \\
\end{array}$ & \begin{tabular}{r|}
10 \\
5 \\
0.254 \\
1.18 \\
1.37 \\
\end{tabular} & \begin{tabular}{r|}
10 \\
5 \\
0.35 \\
1.87 \\
2.40 \\
\end{tabular} & $\begin{array}{r}60 \\
32 \\
0.254 \\
4.16 \\
1.97 \\
\end{array}$ \\
\hline Zinc & $\begin{array}{l}\text { Total Samples } \\
\text { Hits } \\
\text { Minimum Detection } \\
\text { Maximum Delection } \\
\text { Mean } \\
\end{array}$ & $\begin{array}{r}10 \\
6 \\
7.6 \\
14.5 \\
8.55 \\
\end{array}$ & $\begin{array}{r}10 \\
6 \\
7.8 \\
67.3 \\
14.24 \\
\end{array}$ & \begin{tabular}{r|}
10 \\
5 \\
2.83 \\
9.27 \\
3.38 \\
\end{tabular} & $\begin{array}{r}10 \\
4 \\
2.92 \\
7.22 \\
3.18 \\
\end{array}$ & $\begin{array}{r}10 \\
5 \\
3.19 \\
17.3 \\
5.10 \\
\end{array}$ & \begin{tabular}{r|}
10 \\
8 \\
6.39 \\
20.9 \\
10.77 \\
\end{tabular} & $\begin{array}{r}60 \\
34 \\
0 \\
67.3 \\
7.54 \\
\end{array}$ \\
\hline Total Samples & & 190 & 193 & 190 & 190 & 190 & 190 & 1143 \\
\hline Total Hits & & 93 & 87 & 80 & 75 & 80 & 94 & 509 \\
\hline
\end{tabular}


Table 6-2: Background Concentrations for TCL Volatile Organic Compounds, $\mu g / L$

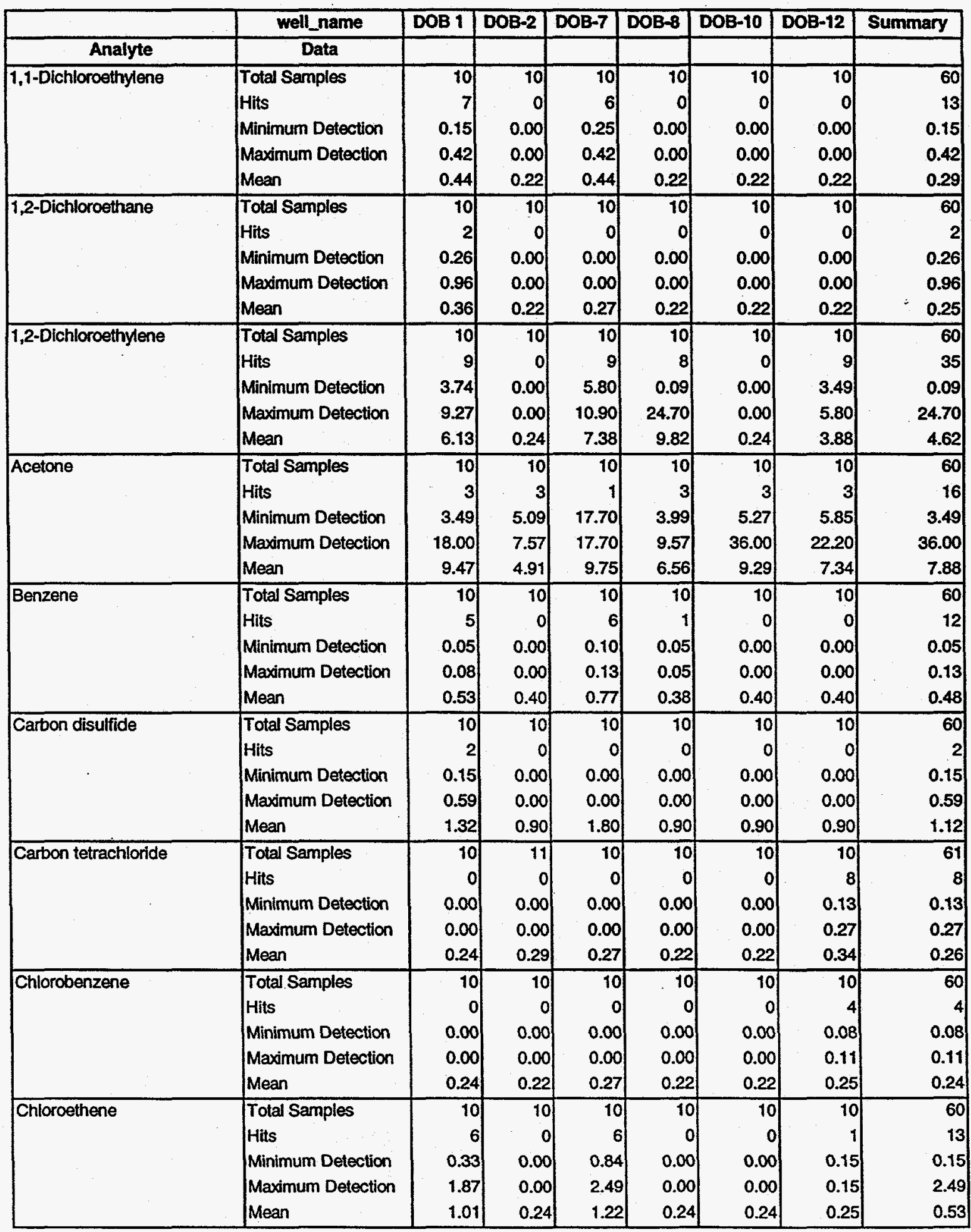


Table 6-2: Background Concentrations for TCL Volatile Organic Compounds, $\mu \mathrm{g} / \mathrm{L}$ (cont'd)

\begin{tabular}{|c|c|c|c|c|c|c|c|c|}
\hline & well_name & DOB 1 & DOB-2 & DOB-7 & DOB-8 & DOB-10 & DOB-12 & Summary \\
\hline Analyte & Data & & & & & & & \\
\hline \multirow[t]{5}{*}{ Chloroform } & Total Samples & 10 & 11 & 10 & 10 & 10 & 10 & 61 \\
\hline & & 이 & 8 & 이 & 이 & 8 & 8) & 24 \\
\hline & Minimum Detection & 0.00 & 0.16 & 0.00 & 0.00 & 0.12 & 0.11 & 0.11 \\
\hline & Maximum Detection & 0.00 & 0.40 . & 0.00 & 0.00 & 0.22 & 0.27 & 0.40 \\
\hline & Mean & 0.24 & 0.48 & 0.27 & 0.22 & 0.34 & 0.33 & 0.32 \\
\hline \multirow[t]{5}{*}{ Dichloromethane } & Total Samples & 10 & 10 & 10 & 10 & 10 & 10 & 60 \\
\hline & & 7 & 6 & 8) & 5 & 6 & 7 & 39 \\
\hline & Minimum Detection & 0.68 & 0.54 & 0.55 & 0.57 & 0.43 & 0.52 & 0.43 \\
\hline & Maximum Detection & 3.60 & 1.19 & 14.80 & 1.34 & 1.26 & 2.64 & 14.80 \\
\hline & Mean & 2.06 & 1.24 & 3.05 & 1.19 & 1.18 & 1.49 & 1.70 \\
\hline \multirow[t]{5}{*}{ Ethylbenzene } & Total Samples & 10 & 10 & 10 & 10 & 10 & 10 & 60 \\
\hline & & o) & 1 & 0 & of & 0 & 1 & \\
\hline & Minimum Detection & 0.00 & 0.16 & 0.00 & 0.00 & 0.00 & 1.63 & 0.16 \\
\hline & Maximum Detection & 0.00 & 0.16 & 0.00 & 0.00 & 0.00 & 1.63 & 1.63 \\
\hline & Mean & 0.24 & 0.23 & 0.27 & 0.22 & 0.22 & 0.28 & 0.24 \\
\hline \multirow[t]{5}{*}{ Methyl ethyl ketone } & Total Samples & 10 & 10 & 10 & 10 & 10 & 10 & 60 \\
\hline & & 1 & 3 & 2 & o) & 2 & 1 & \\
\hline & Minimum Detection & 0.92 & 1.08 & 1.31 & 0.00 & 0.87 & 7.94 & 0.87 \\
\hline & Maximum Detection & 0.92 & 2.48 & 2.80 & 0.00 & 1.54 & 7.94 & 7.94 \\
\hline & Mean & 1.39 & 1.32 & 2.11 & 0.90 & 1.04 & 1.64 & 1.40 \\
\hline \multirow[t]{5}{*}{ Tetrachloroethylene } & Total Samples & 10 & 11 & 10 & 10 & 10 & 10 & 61 \\
\hline & Hits & 10 & 0 & 10 & 9 & 0 & 10 & 39 \\
\hline & Minimum Detection & 5.39 & 0.00 & 8.60 & 0.06 & 0.00 & 0.64 & 0.06 \\
\hline & Maximum Detection & 13.70 & 0.00 & 16.40 & 5.07 & 0.00 & 1.35 & 16.40 \\
\hline & Mean & 8.46 & 0.29 & 12.47 & 2.17 & 0.22 & 0.95 & 4.03 \\
\hline \multirow[t]{5}{*}{ Toluene } & Total Samples & 10 & 10 & 10 & 10 & 10 & 10 & 60 \\
\hline & Hits & 6 & 4 & 6 & 4 & 5 & 7 & \\
\hline & Minimum Detection & 0.07 & 0.07 & 0.07 & 0.07 & 0.05 & 0.06 & 0.05 \\
\hline & Maximum Detection & 0.70 & 1.22 & 0.70 & 0.24 & 0.24 & 4.41 & 4.41 \\
\hline & Mean & 0.38 & 0.44 & 0.63 & 0.35 & 0.33 & 0.66 & 0.46 \\
\hline \multirow[t]{5}{*}{ Trichloroethylene } & Total Samples & 10 & 11 & 10 & 10 & 10 & 10 & 61 \\
\hline & Hits & 10 & 0 & 10 & 6 & of & 10 & \\
\hline & Minimum Detection & 41.80 & 0.00 & 62.30 & 0.13 & 0.00 & 1.83 & 0.1 \\
\hline & Maximum Detection & 87.50 & 0.00 & 118.00 & 0.74 & 0.00 & 3.61 & 118.00 \\
\hline & Mean & 58.72 & 0.29 & 80.96 & 0.48 & 0.22 & 2.40 & 23.46 \\
\hline \multirow[t]{5}{*}{ Xylenes } & Total Samples & 10 & 10 & 10 & 10 & 10 & 10 & 60 \\
\hline & Hits & 1 & 2 & 0 & 2 & 2 & 3 & 10 \\
\hline & Minimum Detection & 0.06 & 0.18 & 0.00 & 0.07 & 0.06 & 0.06 & 0.06 \\
\hline & Maximum Detection & 0.06 & 0.21 & 0.00 & 0.21 & 0.24 & 3.66 & 3.66 \\
\hline & Mean & 0.33 & 0.28 & 0.40 & 0.27 & 0.28 & 0.54 & 0.35 \\
\hline Total Samples & & 170 & 174 & 170 & 170 & 170 & 170 & 1024 \\
\hline Total Hits & & 69 & 27 & 64 & 38 & 26 & 72 & 296 \\
\hline
\end{tabular}


Table 6-3: Background Concentrations for Indicator Parameters, $\mu \mathrm{g} / \mathrm{L}$

\begin{tabular}{|c|c|c|c|c|c|c|c|c|}
\hline & well name & DOB I & DOB 2 & DOB 7 & DOB 8 & DOB 10 & DOB 12 & Summary \\
\hline Analyte & Data & & & & & & & \\
\hline \multirow[t]{5}{*}{ Chemical oxygen demand } & Total Samples & 10 & 10 & 10 & 10 & 10 & 10 & 60 \\
\hline & Hits & 7 & 8 & 9 & 3 & 6 & 3 & 36 \\
\hline & Minimum Detection & 7,530 & 5,270 & 7,600 & 6,280 & 6,110 & 6,110 & 5,270 . \\
\hline & Maximum Detection & 57,300 & 27,200 & 29,700 & 12,600 & 18,600 & 324,000 & 324,000 \\
\hline & Mean & $16,383.00$ & 11,040 & 17,865 & 3,232 & 8,423 & 39,691 & $16,105.67$ \\
\hline \multirow[t]{5}{*}{ Chloride } & Total Samples & 10 & 10 & 10 & 10 & 10 & 10 & 60 \\
\hline & Hits & & & & & & & \\
\hline & Minimum Detection & $1,210.00$ & $4,920.00$ & $1,930.00$ & $1,140.00$ & $3,810.00$ & $5,240.00$ & $1,140.00$ \\
\hline & Maximum Detection & $1,440.00$ & $6,050.00$ & $2,670.00$ & $3,820.00$ & $4,340.00$ & $5,770.00$ & $6,050.00$ \\
\hline & Mean & $1,283.00$ & $5,415.00$ & $2,335.00$ & $2,220.00$ & $3,996.00$ & $5,505.00$ & $3,459.00$ \\
\hline \multirow[t]{5}{*}{ Nitrate-nitrite as nitrogen } & Total Samples & 7 & 7 & 7 & 7 & 7 & 7 & 42 \\
\hline & Hits & 7 & 7 & 7 & 6 & 7 & 7 & 41 \\
\hline & Minimum Detection & 350.00 & 660.00 & 160.00 & 100.00 & 540.00 & $1,040.00$ & 100.00 \\
\hline & Maximum Detection & 800.00 & $1,210.00$ & 530.00 & $1,730.00$ & 1.180 .00 & $1,350.00$ & $1,730.00$ \\
\hline & Mean & 535.71 & 884.29 & 237.14 & 855.71 & 720.00 & $1,237.14$ & 745.00 \\
\hline \multirow[t]{5}{*}{ Nitrate as nitrogen } & Total Samples & 3 & 3 & 3 & 3 & 3 & 3 & 18 \\
\hline & Hits & & 3 & & 3 & 3 & & \\
\hline & Minimum Detection & 797.00 & 617.00 & 128.00 & 33.80 & 562.00 & $1,100.00$ & 33.80 \\
\hline & Maximum Detection & $1,110.00$ & 719.00 & 150.00 & 39.40 & 664.00 & $1,140.00$ & $1,140.00$ \\
\hline & Mean & 972.33 & 657.67 & 142.33 & 36.20 & 623.00 & $1,116.67$ & 591.37 \\
\hline \multirow[t]{5}{*}{$\mathrm{pH}$} & Total Samples & 10 & 10 & 10 & 10 & 10 & 10 & 60 \\
\hline & Hits & 10 & 10 & 10 & 10 & 10 & 10 & 60 \\
\hline & Minimum Detection & 6.20 & 5.04 & 4.70 & 4.92 & 5.17 & 5.33 & 4.70 \\
\hline & Maximum Detection & 6.71 & 5.31 & 5.02 & 7.01 & 5.72 & 5.73 & 7.01 \\
\hline & Mean & 6.43 & 5.19 & 4.91 & 5.24 & 5.45 & 5.56 & 5.46 \\
\hline \multirow[t]{5}{*}{ Specific conductance } & Total Samples & 10 & 10 & 10 & 10 & 10 & 10 & 60 \\
\hline & Hits & 10 & 10 & 10 & 10 & 10 & 10 & 60 \\
\hline & Minimum Detection & 21.60 & 57.30 & 78.50 & 17.20 & 49.00 & 62.40 & 17.20 \\
\hline & Maximum Detection & 142.00 & 70.40 & 94.60 & 56.40 & 59.30 & 70.30 & 142.00 \\
\hline & Mean & 103.06 & 62.59 & 86.03 & 39.32 & 54.07 & 67.54 & 68.77 \\
\hline \multirow[t]{5}{*}{ Sulfate } & Total Samples & 10 & 10 & 10 & 10 & 10 & 10 & 60 \\
\hline & Hits & & 10 & & & & & \\
\hline & Minimum Detection & $11,500.00$ & $9,210.00$ & $21,700.00$ & $5,200.00$ & $6,970.00$ & $6,990.00$ & $5,200.00$ \\
\hline & Maximum Detection & $14,400.00 \mid$ & $11,700.00$ & $28,000.00$ & $8,150.00$ & $9,850.00$ & $9,390.00$ & $28,000.00$ \\
\hline & Mean & $12,800.00$ & $10,443.00$ & $24,000.00$ & $7,064.00$ & $7,943.00$ & $8,376.00$ & $11,771.00$ \\
\hline \multirow[t]{5}{*}{ Total organic carbon } & Total Samples & 10 & 11 & 10 & 10 & 10 & 10 & 61 \\
\hline & Hits & & 11 & & 8 & & & \\
\hline & Minimum Detection & $1,390.00$ & $2,380.00$ & 572.00 & 413.00 & $1,910.00$ & $1,040.00$ & 413.00 \\
\hline & Maximum Detection & $18,800.00$ & $4,150.00$ & $7,980.00$ & $1,660.00$ & $7,590.00$ & $2,630.00$ & $18,800.00$ \\
\hline & Mean & $6,316.00$ & $3,312.73$ & $6,257.20$ & 897.15 & $2,919.00$ & $1,355.00$ & $3,506.29$ \\
\hline \multirow[t]{5}{*}{ Total organic halogens } & Hits & 10 & 11 & 10 & 10 & 10 & 10 & 61 \\
\hline & Minimum Detection & & 10 & & & 8 & 6 & 48 \\
\hline & Maximum Detection & 23.60 & 8.88 & 38.90 & 5.16 & 2.32 & 6.64 & 2.32 \\
\hline & Mean & 54.90 & 86.10 & 76.60 & 56.90 & 96.80 & 21.60 & 96.80 \\
\hline & MEAN & 37.46 & 20.78 & 55.45 & 12.94 & 18.39 & 7.95 & 25.42 \\
\hline Total Samples & & 80 & 82 & 80 & 80 & 80 & 80 & 482 \\
\hline Total Hits & & 77 & 79 & 79 & 64 & 74 & 69 & 442 \\
\hline
\end{tabular}




\subsection{COMPARISON OF POST-IRA SAMPLES TO BACKGROUND VALUES}

Post-IRA samples for the third monitoring event were collected from all six downgradient wells on 12/01/97. The results of the third semi-annual post-IRA sampling are presented on Table 7-1. The maximum concentration for each analyte from each well from the post-IRA samples was then compared to the tolerance interval. The results of the comparison are presented in Table 72. The following interprets the third semi-annual post-IRA sampling results.

\section{TAL Metals}

Aluminum exceeded the tolerance interval in DOB-10. Arsenic exceeded the tolerance interval in DOB-2. Lead was not detected in any sample from any of the wells. Chromium was only detected in DOB-2 at $1.3 \mu \mathrm{g} / \mathrm{L}$ but did not exceed the tolerance interval. Copper concentrations exceeded the tolerance intervals in DOB-1, 2, 7, 8, 10 and 12. Barium concentrations exceeded the tolerance intervals in DOB-7, 10 and 12. Iron concentrations exceeded the tolerance interval in DOB-2, 7, 8 and 10. Magnesium was not detected in any sample from any of the wells. Manganese concentrations exceeded the tolerance interval in DOB-2, 7, 10 and 12. Mercury exceeded its tolerance interval in DOB-2. Zinc also exceeded its tolerance interval in DOB-1, 2, 7 and 10.

Of the 120 tolerance intervals calculated for TAL metals, only 27 (or $19 \%$ ) were exceeded. Conversely, $77.5 \%$ of the TAL metal concentrations were either less than the predicted tolerance interval or were not present at all in groundwater samples. Antimony, cadmium, lead, nickel, selenium, silver, vanadium and thallium, which were all detected at least once during background sampling, disappeared completely from the list of analytes detected in the 4Q97 post-IRA samples. No MCLs were exceeded for any of the TAL metals.

There does not appear to be any consistent pattern or trend for the presence of metal analytes in DOSB groundwater. Concentrations of some of the metals such as arsenic, cadmium, copper, silver, cobalt, lead, mercury, vanadium, zinc and beryllium appear to spike sporadically and then return to levels below detection limits. There appears to be a pattem of random distribution within the wells, indicating that analyte presence may be the result of sampling or measurement error. No long-term trend can be discerned from the data.

\section{TCL Volatiles}

1,2-Dichioroethylene (DCE) exceeded the tolerance interval in DOB-2. Benzene exceeds the tolerance interval in DOB 2 and 10. Chloroethene exceeded the tolerance interval in DOB-2, 10 and 12. Ethylbenzene exceeded the tolerance interval in DOB-10. Tetrachloroethylene (PCE) exceeded the tolerance intervals in DOB-10 $(7.34 \mu \mathrm{g} / \mathrm{L})$ and $12(9.12 \mu \mathrm{g} / \mathrm{L})$. Trichloroethylene (TCE) exceeded the predicted tolerance intervals in DOB-2 $(21.6 \mu \mathrm{g} / \mathrm{L}), \mathrm{DOB}-10(39.1 \mu \mathrm{g} / \mathrm{L})$ and DOB-12 $(8.14 \mu \mathrm{g} / \mathrm{L})$. Xylene exceeded the tolerance interval in DOB-10 $(0.614 \mu \mathrm{g} / \mathrm{L})$.

Of the 204 tolerance intervals calculated for TCL VOCs only 15 (or $7 \%$ ) were exceeded. Conversely, $93 \%$ of the TCL VOC concentrations were either less than the predicted tolerance interval or were not present at all in groundwater samples. 1,1-Dichloroethylene, 1,2dichloroethane, acetone, carbon disulfide, carbon tetrachloride, chloroform, Dichloromethane and methyl ethyl ketone, which were all detected at least once in background sampling, disappeared completely from the list of analytes detected in the 4Q97 post-IRA samples.

The results indicate that Chloroethene (vinyl chloride), has reappeared as a contaminant in groundwater. The maximum concentration was $39.1 \mu \mathrm{g} / \mathrm{L}$ in DOB-10. The reappearance of chloroethene correlates to the shutdown of the bio-vent system. Its presence is interpreted to mean that DOSB has returned to anoxic condition and indigenous bioremediation has resumed. Additionally, the only MCLs exceeded for TCL VOCs was for PCE (MCL=5.0 $\mu \mathrm{g} / \mathrm{L})$ in $\mathrm{DOB}-10$ 
(7.34 $\mu \mathrm{g} / \mathrm{L})$ and DOB-12 $(9.2 \mu \mathrm{g} / \mathrm{L})$ and TCE (MCL $=5.0 \mu \mathrm{g} / \mathrm{L})$ also in DOB-10 (39.1 $\mu \mathrm{g} / \mathrm{L}), \mathrm{DOB}-$ $12(8.14 \mu \mathrm{g} / \mathrm{L})$ and DOB-2 $(21.6 \mu \mathrm{g} / \mathrm{L})$. Dichloromethane, a final CM-COC, was not detected in groundwater at all. The absence of dichloromethane is an indication that the testing of the biovent was successful in destroying this contaminant from basin soils.

\section{Indicator Parameters}

Indicator parameters cannot be interpreted in the same manner as the chemical parameters. By evaluating the relative levels of indicator parameters, it is possible to predict broad changes in the geochemistry and biochemistry of a water system and make general qualitative statements as to the overall nature of the water quality. Depending upon the parameter, a quantitative increase can indicate a positive, negative or neutral trend. Therefore, the following interpretation of indicator parameters will be qualitative.

Chemical oxygen demand (COD) is a measure of the amount of oxygen required to chemically oxidize organics dissolved in the groundwater. COD levels have increased in 3 of the 6, downgradient wells (maximum concentration $37.4 \mathrm{mg} /$ ) but are still relatively low compared to municipal sewage or landfill leachate. Since these levels have increased slightly, it appears that oxygen levels have decreased in the groundwater. The increase in COD levels is related to the discontinuation of the bio-vent since an empirical relationship exists between $C O D$ and $B O D$ (biological oxygen demand) levels.

Groundwater analyses indicate an increase in the levels of chloride in 3 of the downgradient wells. Chloride is produced as an end product of the biodegradation of chlorinated solvents. This indicates a probable combination of aerobic and anaerobic conditions still continuing to degrade the residual chlorinated solvents. The presence of sulfate is attributed to microbial degradation of petroleum compounds. Sulfate concentrations increased significantly in DOB-10 and 12 on the west side of the basin and are interpreted as very positive groundwater quality trends. Nitrates can exist in seven oxidation states in nature which can make nitrate data difficult to interpret. At DOSB the nitrate results are neutral, with two wells showing an increase in nitrate levels and four wells showing a decrease in nitrate levels. The probable explanation for the mixed results is a transition in the groundwater from an aerobic to anaerobic system caused by discontinuing the testing of the bio-vent. Where nitrate levels are low may reflect where locations have greater numbers of aerobic nitrifying bacteria. Where nitrate levels are higher may indicate where anaerobic conditions still prevail and are associated with low dissolved oxygen (DO) levels.

There has been an increase in the total organic carbon concentration that is indicative of the use of organic carbon by microorganisms as a nutrient substrate. This is interpreted as a negative groundwater quality trend and is indicative of the system becoming more anaerobic, thus lowering biodegradation rates. Total Organic Halogen (TOX) levels have increased in 3 of the 6 , downgradient wells. This is also a negative indication that biodegradation rates have slowed digestion of organic halogen compounds in groundwater. As a result, concentrations of PCE can be expected to decrease, while concentrations of VC can be expected to increase because of anaerobic digestion processes will predominate the system.

The results of indicator parameters reflect flux in the DOSB groundwater system again. It is apparent from the results, that the groundwater system is changing from an aerobic system to an anaerobic system and from an oxidizing system to a reducing system. The results are interpreted to indicate that the change in the groundwater system is attributable to the discontinuation of the bio-vent testing. Further chemical evidence is available to support this hypothesis. Table 7-3 illustrates a comparison of the pre-IRA maximum concentration of each analyte by well to the post-IRA maximum concentration of each analyte by well. The results are important in that the reappearance of chloroethene is directly related to the increase in anaerobic degradation within the system, especially on the west side of the basin which was the most contaminated portion of DOSB. Generally, PCE concentrations have decreased which is also 
indicative of increased anaerobic conditions within the system. COD and total organic carbon (TOC) levels have also increased in three of the downgradient wells also located on the west side of the basin and is clear evidence of the impact of the discontinuation of the bio-vent. Because biodegradation rates are slower under anaerobic conditions the rate of digestion of TOC will also be slower. There still appears to be significant indigenous anaerobic microbial degradation occurring since chloride levels have significantly increased indicating destruction of chlorinated solvents.

\subsection{CONCLUSION}

It is concluded from evaluation of the chemical data, from the time of the IRA to the 4Q97 postIRA samples, that there is no significant evidence of negative impact or release of hazardous substances to the groundwater caused by the IRA. Based upon the chemical data, it appears that groundwater quality is still significantly improved since the time of the IRA. This is evidenced by the fact that there were no MCL exceedances for any of the TAL metals and the complete disappearance of dichloromethane, a $\mathrm{CM}-\mathrm{COC}$, in groundwater. Maximum concentrations of other organic compounds, such as TCE and PCE, have significantly decreased since the time of the IRA. The maximum concentration of $4 \mathrm{Q} 97$ samples of PCE compared to pre-IRA samples has decreased from $16.4 \mu \mathrm{g} / \mathrm{L}$ to $9.12 \mu \mathrm{g} / \mathrm{L}$ and the maximum concentration of TCE has decreased from $118 \mu \mathrm{g} / \mathrm{L}$ to $39.1 \mu \mathrm{g} / \mathrm{L}$.

The only compound that counters this trend is chloroethene (VC). VC was not detected in any of the 2Q97 groundwater samples while the bio-vent was being testing. VC was not disposed in the DOSB and its presence is attributable to the anaerobic degradation of TCE and PCE. Under anaerobic conditions PCE is degraded and concentrations will decrease based upon first-order biodegradation rates. The degradation chain then becomes PCE-TCE-DCE-VC. This is also referred to as reductive dechlorination. Under aerobic conditions PCE is not degraded and concentrations remain constant. The degradation chain then becomes TCE-DCE-ethylene.

Since bio-vent testing was discontinued, VC has reappeared as a contaminant in groundwater (maximum concentration of $47.2 \mu \mathrm{g} / \mathrm{L}$ in DOB-10). The production of VC at DOSB is a predictable biochemical process caused by reductive dechlorination under anoxic conditions. Since the production rates for VC under anoxic conditions is low, and the destruction rates for VC were high during bio-vent testing, VC disappeared. Since bio-vent testing has been terminated, VC concentrations will return and remain persistent under anoxic conditions.

However, DOSB still evidences indigenous anaerobic biodegradation and overall groundwater quality should improve with time. 


\begin{tabular}{|c|c|c|c|c|c|c|c|c|}
\hline & & well_name & & & & & & \\
\hline \multirow{2}{*}{\begin{tabular}{|l} 
analyte, name \\
1,2-Dichloroethylene
\end{tabular}} & Data & DOB 1 & DOB 2 & DOB 7 & DOB 8 & DOB 10 & DOB 12 & Grand Total \\
\hline & $\begin{array}{l}\text { Total Detections } \\
\text { Maximum Concentration }\end{array}$ & $\mathrm{ND}$ & $\begin{array}{r}11.7 \\
11.7\end{array}$ & $\mathrm{ND}$ & ND & ND & ND & 11.7] \\
\hline Aluminum, total recoverable & $\begin{array}{l}\text { Total Detections } \\
\text { Maximum Concentration }\end{array}$ & $\begin{array}{r}1 \\
118 \\
\end{array}$ & $\begin{array}{r}1 \\
15.8 \\
\end{array}$ & $\begin{array}{r}1 \\
66.8 \\
\end{array}$ & ND & $\begin{array}{r}1 \\
6930 \\
\end{array}$ & ND & $\begin{array}{r}4 \\
6930 \\
\end{array}$ \\
\hline Arsenic, total recoverable & $\begin{array}{l}\text { Total Detections } \\
\text { Maximum Concentration }\end{array}$ & ND & $\begin{array}{r}1 \\
4.2 \\
\end{array}$ & $\mathrm{ND}$ & ND & $\mathrm{ND}$ & ND & $\begin{array}{r}1 \\
4.2 \\
\end{array}$ \\
\hline Barium, total recoverable & $\begin{array}{l}\text { Total Detections } \\
\text { Maximum Concentration }\end{array}$ & $\begin{array}{r}1 \\
7.48 \\
\end{array}$ & $\begin{array}{r}1 \\
29 \\
\end{array}$ & $\begin{array}{r}1 \\
56.9 \\
\end{array}$ & $\begin{array}{r}1 \\
10.6 \\
\end{array}$ & \begin{tabular}{r|}
1 \\
107 \\
\end{tabular} & \begin{tabular}{r|}
1 \\
31.4 \\
\end{tabular} & $\begin{array}{r}6 \\
107 \\
\end{array}$ \\
\hline Benzene & $\begin{array}{l}\text { Total Detections } \\
\text { Maximum Concentration }\end{array}$ & $\mathrm{ND}$ & $\begin{array}{r}1 \\
0.266 \\
\end{array}$ & $\mathrm{ND}$ & ND & $\begin{array}{r}1 \\
1.44 \\
\end{array}$ & ND & $\begin{array}{r}2 \\
1.44 \\
\end{array}$ \\
\hline Beryllium, total recoverable & $\begin{array}{l}\text { Total Detections } \\
\text { Maximum Concentration }\end{array}$ & $\mathrm{ND}$ & ND & $\mathrm{ND}$ & ND & $\begin{array}{r}1 \\
0.64\end{array}$ & $\mathrm{ND}$ & \\
\hline Calcium, total recoverable & $\begin{array}{l}\text { Total Detections } \\
\text { Maximum Concentration }\end{array}$ & $\begin{array}{r}1 \\
3360 \\
\end{array}$ & $\begin{array}{r}1 \\
2800 \\
\end{array}$ & $\begin{array}{r}1 \\
3490 \\
\end{array}$ & $\begin{array}{r}1 \\
1450 \\
\end{array}$ & $\begin{array}{r}1 \\
19000 \\
\end{array}$ & $\begin{array}{r}1 \\
4060 \\
\end{array}$ & $\begin{array}{r}6 \\
19000 \\
\end{array}$ \\
\hline Chemical oxygen demand & \begin{tabular}{|l|} 
Total Detections \\
Maximum Concentration
\end{tabular} & $\begin{array}{r}1 \\
10000 \\
\end{array}$ & $\begin{array}{r}1 \\
37400 \\
\end{array}$ & $\begin{array}{r}1 \\
13000 \\
\end{array}$ & ND & $\begin{array}{r}1 \\
28000 \\
\end{array}$ & $\begin{array}{r}1 \\
10000 \\
\end{array}$ & $\begin{array}{r}5 \\
37400 \\
\end{array}$ \\
\hline Chloride & $\begin{array}{l}\text { Total Delections } \\
\text { Maximum Concentration }\end{array}$ & $\begin{array}{r}1 \\
1320 \\
\end{array}$ & $\begin{array}{r}1 \\
4180 \\
\end{array}$ & $\begin{array}{r}1 \\
2830 \\
\end{array}$ & $\begin{array}{r}1 \\
672 \\
\end{array}$ & $\begin{array}{r}1 \\
5240 \\
\end{array}$ & $\begin{array}{r}1 \\
6600 \\
\end{array}$ & $\begin{array}{r}6 \\
6600 \\
\end{array}$ \\
\hline Chlorobenzene & $\begin{array}{l}\text { Total Detections } \\
\text { Maximum Concentration }\end{array}$ & $\mathrm{ND}$ & $\begin{array}{r}1 \\
1.1 \\
\end{array}$ & $\mathrm{ND}$ & ND & ND & $\begin{array}{r}1 \\
0.412 \\
\end{array}$ & $\begin{array}{r}2 \\
1.1 \\
\end{array}$ \\
\hline Chloroethene (Vinyl chloride) & $\begin{array}{l}\text { Total Detections } \\
\text { Maximum Concentration }\end{array}$ & $\mathrm{ND}$ & $\begin{array}{r}1 \\
2.2 \\
\end{array}$ & $\mathrm{ND}$ & ND & $\begin{array}{r}1 \\
47.2 \\
\end{array}$ & $\begin{array}{r}1 \\
2.29 \\
\end{array}$ & $\begin{array}{r}3 \\
47.2 \\
\end{array}$ \\
\hline Chromium, total recoverable & $\begin{array}{l}\text { Total Detections } \\
\text { Maximum Concentration }\end{array}$ & $\mathrm{ND}$ & $\begin{array}{r}1 \\
1.3 \\
\end{array}$ & $\mathrm{ND}$ & ND & ND & $\mathrm{ND}$ & $\begin{array}{r}1 \\
1.3 \\
\end{array}$ \\
\hline Cobalt, total recoverable & $\begin{array}{l}\text { Total Detections } \\
\text { Maximum Concentration }\end{array}$ & ND & $\mathrm{ND}$ & $\mathrm{ND}$ & ND & $\begin{array}{r}1 \\
7.15 \\
\end{array}$ & ND & $\begin{array}{r}1 \\
7.15 \\
\end{array}$ \\
\hline Copper, total recoverable & $\begin{array}{l}\text { Total Detections } \\
\text { Maximum Concentration }\end{array}$ & $\begin{array}{r}1 \\
84.3 \\
\end{array}$ & $\begin{array}{r}1 \\
6.83 \\
\end{array}$ & $\begin{array}{r}1 \\
2.76 \\
\end{array}$ & $\begin{array}{r}1 \\
6.18 \\
\end{array}$ & $\begin{array}{r}1 \\
6.19 \\
\end{array}$ & $\begin{array}{r}1 \\
14.5 \\
\end{array}$ & $\begin{array}{r}6 \\
84.3 \\
\end{array}$ \\
\hline Ethylbenzene & $\begin{array}{l}\text { Total Detections } \\
\text { Maximum Concentration }\end{array}$ & $\mathrm{ND}$ & $\mathrm{ND}$ & $\mathrm{ND}$ & ND & $\begin{array}{r}1 \\
0.202 \\
\end{array}$ & ND & $\begin{array}{r}1 \\
0.202 \\
\end{array}$ \\
\hline iron, total recoverable & $\begin{array}{l}\text { Total Detections } \\
\text { Maximum Concentration }\end{array}$ & $\begin{array}{r}1 \\
90.7 \\
\end{array}$ & $\begin{array}{r}1 \\
339 \\
\end{array}$ & $\begin{array}{r}1 \\
219 \\
\end{array}$ & $\begin{array}{r}1 \\
117 \\
\end{array}$ & $\begin{array}{r}1 \\
23300 \\
\end{array}$ & ND & $\begin{array}{r}5 \\
23300 \\
\end{array}$ \\
\hline Manganese, total recoverable & $\begin{array}{l}\text { Total Detections } \\
\text { Maximum Concentration }\end{array}$ & $\begin{array}{r}1 \\
2.58 \\
\end{array}$ & $\begin{array}{r}1 \\
13.1 \\
\end{array}$ & $\begin{array}{r}1 \\
172 \\
\end{array}$ & $\begin{array}{r}1 \\
2.67 \\
\end{array}$ & $\begin{array}{r}1 \\
669 \\
\end{array}$ & $\begin{array}{r}1 \\
107 \\
\end{array}$ & $\begin{array}{r}6 \\
669 \\
\end{array}$ \\
\hline Mercury, total recoverable & $\begin{array}{l}\text { Total Detections } \\
\text { Maximum Concentration }\end{array}$ & ND & $\begin{array}{r}1 \\
0.216 \\
\end{array}$ & $\mathrm{ND}$ & ND & ND & $\mathrm{ND}$ & $\begin{array}{r}1 \\
0.216 \\
\end{array}$ \\
\hline Nickel, total recoverable & $\begin{array}{l}\text { Total Detections } \\
\text { Maximum Concentration }\end{array}$ & ND & $\begin{array}{r}1 \\
2.9\end{array}$ & ND & ND & $\begin{array}{r}1 \\
28.3 \\
\end{array}$ & $\mathrm{ND}$ & \begin{tabular}{r|}
28 \\
28.3 \\
\end{tabular} \\
\hline Nitrate-nitrite as nitrogen & $\begin{array}{l}\text { ns } \\
\text { ncentration }\end{array}$ & $\begin{array}{r}1 \\
726 \\
\end{array}$ & $\begin{array}{r}1 \\
805 \\
\end{array}$ & $\begin{array}{r}1 \\
349 \\
\end{array}$ & ND & ND & $\begin{array}{r}1 \\
946 \\
\end{array}$ & $\begin{array}{r}4 \\
946 \\
\end{array}$ \\
\hline $\mathrm{pH}$ & $\begin{array}{l}\text { Total Detections } \\
\text { Maximum Concentration }\end{array}$ & $\begin{array}{r}1 \\
6.7 \\
\end{array}$ & $\begin{array}{r}1 \\
5.2 \\
\end{array}$ & $\begin{array}{r}1 \\
5.1 \\
\end{array}$ & $\begin{array}{r}1 \\
5.2 \\
\end{array}$ & $\begin{array}{r}1 \\
4.4 \\
\end{array}$ & $\begin{array}{r}1 \\
5.5 \\
\end{array}$ & $\begin{array}{r}6 \\
6.7 \\
\end{array}$ \\
\hline Potassium, total recoverable & $\begin{array}{l}\text { Total Detections } \\
\text { Maximum Concentration }\end{array}$ & $\begin{array}{r}1 \\
3220 \\
\end{array}$ & $\begin{array}{r}1 \\
2230 \\
\end{array}$ & $\begin{array}{r}1 \\
3770 \\
\end{array}$ & $\begin{array}{r}1 \\
2040 \\
\end{array}$ & $\begin{array}{r}1 \\
3670 \\
\end{array}$ & $\begin{array}{r}1 \\
3020 \\
\end{array}$ & $\begin{array}{r}6 \\
3770 \\
\end{array}$ \\
\hline Sodium, total recoverable & $\begin{array}{l}\text { Tolal Detections } \\
\text { Maximum Concentration }\end{array}$ & 860 & $\begin{array}{r}1 \\
2370 \\
\end{array}$ & $\begin{array}{r}1 \\
2450 \\
\end{array}$ & $\begin{array}{r}1 \\
567 \\
\end{array}$ & $\begin{array}{r}1 \\
6070 \\
\end{array}$ & $\begin{array}{r}1 \\
5640 \\
\end{array}$ & $\begin{array}{r}6 \\
6070 \\
\end{array}$ \\
\hline Specific conductance & $\begin{array}{l}\text { Total Detections } \\
\text { Maximum Concentration }\end{array}$ & $\begin{array}{r}1 \\
78 \\
\end{array}$ & $\begin{array}{r}1 \\
49 \\
\end{array}$ & $\begin{array}{r}1 \\
80 \\
\end{array}$ & $\mathrm{ND}$ & $\begin{array}{r}1 \\
419 \\
\end{array}$ & $\begin{array}{r}1 \\
78 \\
\end{array}$ & $\begin{array}{r}5 \\
419 \\
\end{array}$ \\
\hline Sulfate & $\begin{array}{l}\text { Total Detections } \\
\text { Maximum Concentration }\end{array}$ & $\begin{array}{r}1 \\
7200 \\
\end{array}$ & $\begin{array}{r}1 \\
7970 \\
\end{array}$ & $\begin{array}{r}1 \\
25100 \\
\end{array}$ & $\begin{array}{r}1 \\
5850 \\
\end{array}$ & $\begin{array}{r}1 \\
188000 \\
\end{array}$ & $\begin{array}{r}1 \\
12300 \\
\end{array}$ & $\begin{array}{r}6 \\
188000 \\
\end{array}$ \\
\hline Tetrachloroethylene & $\begin{array}{l}\text { Total Detections } \\
\text { Maximum Concentration }\end{array}$ & $\begin{array}{r}1 \\
0.495 \\
\end{array}$ & $\begin{array}{r}1 \\
2.59 \\
\end{array}$ & $\begin{array}{r}1 \\
3.02 \\
\end{array}$ & ND & $\begin{array}{r}1 \\
7.34 \\
\end{array}$ & $\begin{array}{r}1 \\
9.12 \\
\end{array}$ & $\begin{array}{r}5 \\
9.12 \\
\end{array}$ \\
\hline Toluene & $\begin{array}{l}\text { Total Detections } \\
\text { Maximum Concentration }\end{array}$ & ND & ND & ND & $\mathrm{ND}$ & $\begin{array}{r}1 \\
0.208 \\
\end{array}$ & ND & $\begin{array}{r}1 \\
0.208 \\
\end{array}$ \\
\hline Total organic carbon & $\begin{array}{l}\text { Total Detections } \\
\text { Maximum Concentration }\end{array}$ & ND & $\begin{array}{r}1 \\
10000 \\
\end{array}$ & $\begin{array}{r}1 \\
5000 \\
\end{array}$ & $\mathrm{ND}$ & $\begin{array}{r}1 \\
16000 \\
\end{array}$ & $\begin{array}{r}1 \\
9000 \\
\end{array}$ & $\begin{array}{r}4 \\
16000 \\
\end{array}$ \\
\hline Total organic halogens & $\begin{array}{l}\text { Total Detections } \\
\text { Maximum Concentration }\end{array}$ & $\begin{array}{r}1 \\
26.3 \\
\end{array}$ & $\begin{array}{r}1 \\
44.3 \\
\end{array}$ & $\begin{array}{r}1 \\
19.7 \\
\end{array}$ & $\begin{array}{r}1 \\
9.74 \\
\end{array}$ & $\begin{array}{r}1 \\
210 \\
\end{array}$ & $\begin{array}{r}1 \\
38.4 \\
\end{array}$ & $\begin{array}{r}6 \\
210 \\
\end{array}$ \\
\hline Trichloroethylene & $\begin{array}{l}\text { Total Detections } \\
\text { Maximum Concentration }\end{array}$ & $\begin{array}{r}1 \\
0.762 \\
\end{array}$ & $\begin{array}{r}1 \\
21.6 \\
\end{array}$ & $\begin{array}{r}1 \\
3.96 \\
\end{array}$ & $\mathrm{ND}$ & $\begin{array}{r}1 \\
39.1 \\
\end{array}$ & $\begin{array}{r}1 \\
8.14 \\
\end{array}$ & $\begin{array}{r}5 \\
39.1 \\
\end{array}$ \\
\hline Xylenes & \begin{tabular}{|l|} 
Total Detections \\
Maximum Concentration
\end{tabular} & ND & $\mathrm{ND}$ & ND & ND & $\begin{array}{r}1 \\
0.614 \\
\end{array}$ & $\mathrm{ND}$ & 0.614 \\
\hline Zinc, total recoverable & $\begin{array}{l}\text { Total Detections } \\
\text { Maximum Concentration }\end{array}$ & $\begin{array}{r}1 \\
33.5 \\
\end{array}$ & $\begin{array}{r}1 \\
27.8 \\
\end{array}$ & $\begin{array}{r}1 \\
22.5 \\
\end{array}$ & $\mathrm{ND}$ & $\begin{array}{r}1 \\
107 \\
\end{array}$ & $\begin{array}{r}12.4 \\
\end{array}$ & $\begin{array}{r}5 \\
107 \\
\end{array}$ \\
\hline Total Detections & & 18 & 27 & 19 & 11 & 26 & 19 & 120 \\
\hline
\end{tabular}


TABLE 7-2: COMPARISON OF POST ARA SAMPLES TO PREDICTED TOLERANCE WTERVALS, pgh

\begin{tabular}{|c|c|c|c|c|c|c|c|c|c|c|c|c|}
\hline \multirow{2}{*}{$\begin{array}{l}\text { ANALYTE } \\
\text { Volditie Organic Compounds, ugh }\end{array}$} & \multicolumn{2}{|c|}{ DOB1 } & \multicolumn{2}{|c|}{ DOB2 } & \multicolumn{2}{|c|}{ DOB 7} & \multicolumn{2}{|c|}{ DOB 8} & \multicolumn{2}{|c|}{ DOB 10} & \multicolumn{2}{|c|}{ DOB 12} \\
\hline & Max: & S.UCd & $\operatorname{Max}$ & $95 . \mathrm{UCL}$ & $\operatorname{Max}$ & 95UCL & Max & \$5 घCL & Max & SDUC: & $\max$ & $\$ 50 C L$ \\
\hline 1,1,1-Trichloroethane & ND & ND & NO & ND & $\mathrm{ND}$ & ND & ND & ND & ND & ND & ND| & ND \\
\hline 1,1,2,2-Tetrachloroethane & ND & $\mathrm{ND}$ & $\mathrm{ND}$ & ND & ND & ND & ND & $\mathrm{ND}$ & ND & ND| & ND) & ND \\
\hline 1,1,2-Trichloroethane & ND & ND & ND & ND & ND & ND & ND & $\mathrm{ND}$ & ND & ND & ND & ND \\
\hline 1,1-Dichloroethane & ND & ND & ND & ND & $\mathrm{ND}$ & ND & ND & $\mathrm{ND}$ & ND & ND & ND & ND \\
\hline 1,1-Dichloroethylene & ND & 0.61 & $\mathrm{ND}$ & ND & ND| & 0.60 & ND & ND| & ND & ND| & ND & ND \\
\hline 1,2-Dichloroethane & ND & 0.60 & $\mathrm{ND}$ & ND & ND & ND & ND & $\mathrm{ND}$ & ND & ND & ND & ND \\
\hline 1,2-Dichloroethylene & ND & 7.56 & 11.7 & ND & ND & 9.00 & ND & 15.29 & ND & ND & ND & 4.60 \\
\hline 1.2-Dichloropropane & ND & ND & ND| & ND & ND & ND & ND & ND & ND & ND & ND & ND \\
\hline 2-Hexanone & ND & ND & $\mathrm{ND}$ & ND & ND & ND & ND & ND & ND & ND| & ND & ND \\
\hline Acetone & ND & 14.58 & ND| & 6.47 & ND & 16.23 & ND & 8.93 & ND & 16.35 & ND & 11.75 \\
\hline Benzene & ND & 0.96 & 0.266 & ND & ND & 1.31 & ND & 0.56 & 1.44 & ND & ND & ND \\
\hline Bromodichloromethane & ND & ND & ND & ND & ND & ND & ND & $\mathrm{ND}$ & ND & ND & ND & ND \\
\hline Bromoform & ND| & ND| & ND & ND & ND & $\mathrm{ND}$ & ND & ND & ND & ND & ND & ND \\
\hline Bromomethane & ND & ND & $\mathrm{ND}$ & ND & ND & ND & ND & ND & ND & ND| & ND & ND \\
\hline Carbon disulfide & ND & 2.18 & ND & ND & ND & $\mathrm{ND}$ & ND & ND & ND & ND & ND & ND \\
\hline Carbon tetrachloride & ND & ND & ND & ND & ND & $\mathrm{ND}$ & ND & ND & ND & ND) & ND & 0.54 \\
\hline Chbrobenzene & ND & ND| & 1.1 & ND & ND & ND & ND & ND & ND & ND| & 0.412 & 0.47 \\
\hline Chloroethane & ND| & ND & $\mathrm{ND}$ & ND & ND & ND & ND & ND & ND & ND) & ND & ND \\
\hline Chloroethene & ND & 1.34 & 2.2 & ND & ND & 1.55 & ND & $\mathrm{ND}$ & 47.2 & ND & 2.29 & 0.47 \\
\hline Chloroform & ND| & ND & ND & 0.66 & ND & ND & ND & $\mathrm{ND}$ & ND & 0.53 & ND & 0.53 \\
\hline Chloromethane & ND & ND| & ND| & ND & ND & ND & ND & $\mathrm{ND}$ & ND & ND & ND & ND \\
\hline cis-1,3-Dichloropropene & ND| & ND & ND & ND & ND & ND & ND & $\mathrm{ND}$ & ND & ND| & No & ND \\
\hline Dibromochloromethane & ND & ND & ND & ND & $\mathrm{ND}$ & $\mathrm{ND}$ & ND & $\mathrm{ND}$ & ND & ND & ND & ND \\
\hline Dichloromethane & ND & 3.03 & $\mathrm{ND}$ & 1.99 & ND & 5.47 & ND & 1.96 & ND & 1.93 & ND & 2.24 \\
\hline Ethylbenzene & ND & ND & ND & 0.46 & ND & ND & ND & ND| & 0.202 & $\mathrm{ND}$ & ND & 0.59 \\
\hline Melhyl ethyl ketone & ND & 2.22 & ND & 1.84 & ND & 3.09 & ND & $\mathrm{ND}$ & ND & 1.50 & ND & 2.94 \\
\hline Methyl isobutyl ketone & ND & ND & ND & ND & ND & ND & ND & ND & ND & $\mathrm{ND}$ & ND & ND \\
\hline Styrene & ND & ND & ND & $\mathrm{ND}$ & ND & ND & ND & ND| & ND & $\mathrm{ND}$ & ND| & ND \\
\hline Tetrachloroethylene & 0.495 & 10.08 & 2.59 & $\mathrm{ND}$ & 3.02 & 13.85 & ND & 3.13 & 7.34 & ND & 9.12 & 1.10 \\
\hline Toluene & ND & 0.59 & ND & 0.64 & ND & 1.04 & ND & 0.54 & 0.208 & 0.53 & $\mathrm{ND}$ & 1.40 \\
\hline trans-1,3-Dichloropropene & ND & ND & ND & ND & ND & ND & ND & ND & ND & ND & ND & ND \\
\hline Trichloroethylene & 0.762 & 66.39 & 21.6 & ND & 3.96 & 90.14 & ND & 0.68 & 39.1 & ND & 8.14 & 2.70 \\
\hline Vinyl acetate & ND & ND) & ND & ND & $\mathrm{ND}$ & ND & ND & ND & ND & ND & $\mathrm{ND}$ & ND \\
\hline Xylenes & $\mathrm{ND}$ & 0.55 & ND & 0.49 & ND & ND & ND & 0.48 & 0.614 & 0.49 & $\mathrm{ND}$ & 1.16 \\
\hline Metals; ug $/$ & Max & 95.UCL & $\operatorname{Max}$ & \$5UCL & Max & $954 a$ & Max & $95 \mathrm{UCl}$ & Max & \$5 पCL & Max & S5UC4 \\
\hline Aluminum & 118 & 340.02 & 15.8 & 55.37 & 66.8 & 107.17 & ND & 99.68 & 6930 & 650.92 & NDI & 81.76 \\
\hline Antimony & ND & 2.53 & ND & $\mathrm{ND}$ & ND & ND & ND & ND| & ND & $\mathrm{ND}$ & ND & ND \\
\hline Arsenic & ND & 2.53 & 4.2 & 2.54 & ND & ND & ND & $\mathrm{ND}$ & ND & 2.54 & NO & ND \\
\hline Barium & 7.48 & 15.33 & 29 & 29.50 & 56.9 & 48.89 & 10.6 & 21.09 & 107 & 23.17| & 31.4 & 20.09 \\
\hline Berylitium & $\mathrm{ND}$ & ND & ND & 0.67 & ND| & ND & ND & ND & 0.64 & $\mathrm{ND}$ & $N D$ & 0.72 \\
\hline Cadmium & ND & 1.07 & ND & 1.07 & ND & 0.64 & $\mathrm{ND}$ & 1.05 & ND & ND & NDI & ND \\
\hline Chromium & ND & 2.03 & 1.3 & 1.96 & ND & 1.99 & ND & 1.83 & ND & 3.16 & ND: & 1.92 \\
\hline Caball & ND & 1.65 & ND| & 1.10 & ND & 1.12 & ND & 1.05 & 7.15 & 124 & ND & 1.06 \\
\hline Copper & 84.3 & 62.54 & 6.83 & 2.37 & 2.76 & 1.76 & 6.18 & 2.02 & 6.19 & 7.40 & 14.5 & 5.16 \\
\hline Iron & 90.7 & 374.77 & 339 & 236.36 & 219 & 20.82 & 117 & 49.59| & 23300 & 197.01 & ND & 78.42 \\
\hline Lead & ND & 3.37 & ND & 2.55 & ND| & 2.98 & ND & ND| & ND & 7.42 & ND & ND \\
\hline Magnesium & ND & 9478 & ND & 1998 & ND & 4293 & ND & 822 & ND & 2110 & ND & 903.27 \\
\hline Manganese & 2.58 & 5.83 & 13.1 & 14.75 & 172 & 164.39 & 2.67 & 6.09 & 669 & 11.02 & 107 & 6.11 \\
\hline Mercury & ND| & 0.08 & 0.216 & 0.17 & ND & 0.14 & ND & 0.16 & ND & 0.12 & ND| & 0.10 \\
\hline Nickel & ND & 4.88 & 2.9 & 4.37 & $\mathrm{ND}$ & 4.86 & ND & 5.28 & 28.3 & 5.30 & ND & 3.08 \\
\hline Selenium & ND & 2.63 & ND & ND & ND & $\mathrm{ND}$ & $\mathrm{ND}$ & 2.55 & ND & ND & ND & ND \\
\hline Silver & ND & ND & ND & ND & ND & $\mathrm{ND}$ & ND & ND & ND & ND & ND & ND \\
\hline Thallium & ND & ND & ND & $\mathrm{ND}$ & ND & $\mathrm{ND}$ & ND & 2.50 & ND & ND & ND & ND \\
\hline Vanadium & ND & 2.98 & ND & 3.03 & $\mathrm{ND}$ & 4.45 & ND & 1.66 & ND & 2.44 & ND & 3.66 \\
\hline Zinc & 33.5 & 10.57 & 27.8 & 24.89 & 22.5 & 4.59 & ND & 4.10 & ร0? & 7.69 & 12.4 & 13.92 \\
\hline Indicator Parameters, ug $h$ & $\operatorname{Max}$ & $95 \mathrm{VCL}$ & Max & 95 UC: & Max & $95 \mathrm{UCL}$ & $\operatorname{Max}$ & $95 \mathrm{UCL}$ & Max & $95 \mathrm{UCl}$ & Max & $95 \mathrm{UCL}$ \\
\hline Chemical oxygen demand & 10000 & 25567.97 & 37400 & 16299.16 & 13000 & 22031.56 & ND & 5787.01 & 28000 & 12036.07 & 10000 & 95670.54 \\
\hline Chioride & 1320 & 1323.16 & 4180 & 5649.25 & 2830 & 2487.88 & 672 & 2817.41 & 5240 & 4097.57 & 6600 & 5621.40 \\
\hline Nitrate as nitrogen & ND| & 663.51 & ND & 1030.47 & ND & 326.55 & ND & 1370.02 & ND & 866.72 & $\mathrm{ND}$ & 1321.49 \\
\hline Nitrate-nitrite as nitrogen & 726 & 1149.66 & 805 & 717.62 & 349 & 156.11 & ND & 39.40 & ND & 682.74 & 946 & 1139.76 \\
\hline $\mathrm{pH}$ (pH units) & 6.7 & $6.34-6.53$ & 5.2 & $5.14-5.24$ & 5.1 & $4.84-4.98$ & 52 & $4.81-5.66$ & 4.4 & $5.35-5.54$ & 5.5 & $|5.48-5.64|$ \\
\hline Specific conductance (USCM) & 78 & 121.13 & 49 & 64.72 & 80 & 88.62 & ND & 46.22 & 419 & 55.91 & 78 & 69.10 \\
\hline Sulfate & 7200 & 13360.19 & 7970 & 10949.61 & 25100 & 25154.94 & 5850 & 7628.64 & 188000 & 8480.19 & 12300 & 8788.75 \\
\hline Total organic carbon & 7000 & 8853.21 & 10000 & 3626.07 & 5000 & 7388.66 & ND & 1140.75 & 16000 & 3846.49| & 9000 & 1611.22 \\
\hline Tofal organic halogens & 26.3 & 44.07 & 44.3 & 32.71 & 19.7 & 61.39 & 9.74 & 22.13 & 210 & 33.95 & 38.4 & 10.81 \\
\hline
\end{tabular}


THIRD SEMI-ANNUAL GROUNDWATER MONITORING

WSRC-RP-98-4110

REPORT FOR THE INTERIM REMEDIAL ACTION

JUNE 1998

D-AREA OIL SEEPAGE BASIN

PAGE 17 OF 17

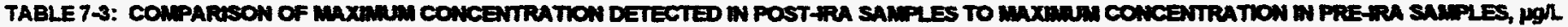

\begin{tabular}{|c|c|c|c|c|c|c|c|c|c|c|c|c|}
\hline 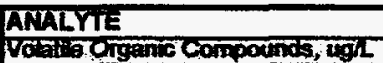 & $\begin{array}{l}\text { DOE } \\
\text { POAमIRAI }\end{array}$ & PTETA & 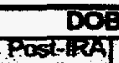 & 2 & DOS & 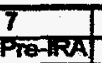 & 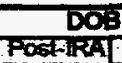 & 8 & DOS & Fo & 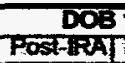 & T2 \\
\hline 1,1,1-Trichloroethane & ND & ND! & ND & ND & ND & ND & ND & ND & ND & ND| & NDI & NO \\
\hline $1,1,2,2-$-Tetrachloroethane & ND & ND) & ND & ND & ND & ND & ND & ND & $\mathrm{ND}$ & ND & ND & ND \\
\hline 1,1,2-Trichloroethane & ND| & $\mathrm{ND}$ & ND & ND & ND| & ND & ND & ND & ND) & ND| & ND & ND \\
\hline 1,1-Dichloroethane & ND & $\mathrm{ND}$ & ND & ND & ND| & ND & ND & ND & ND & ND| & ND & ND \\
\hline 1,1-Dichloroethylene & ND & 0.42 & ND & ND & ND & 0.42 & ND & ND & ND & 0.45 & ND & 0.45 \\
\hline 1,2-Dichloroethane & $\mathrm{ND}$ & 0.96 & ND & ND & ND & ND & ND & ND & ND| & ND & ND & ND \\
\hline 1,2-Dichloroethylene & $\mathrm{ND}$ & 9.27 & $\$ 1.7$ & ND & ND & 10.90 & ND & 24.70 & ND| & ND & $\mathrm{ND}$ & 5.80 \\
\hline 1,2-Dichloropropane & NO & $\mathrm{ND}$ & $\mathrm{ND}$ & ND & ND & ND & ND & ND & ND & $\mathrm{ND}$ & ND & ND \\
\hline 2-Hexanone & ND| & ND & ND) & ND & ND| & ND & ND & ND & $\mathrm{ND}$ & $\mathrm{ND}$ & ND & ND \\
\hline Acetone & ND & 18.00 & ND & 7.57 & ND & 17.70 & ND & 9.57| & ND & 36.00 & $\mathrm{ND}$ & 22.20 \\
\hline Benzene & ND & 0.08 & 0.226 & ND & ND & 0.13 & ND & 0.05 & 1.44 & $\mathrm{ND}$ & $\mathrm{ND}$ & $\mathrm{ND}$ \\
\hline Bromodichloromethane & ND| & ND & ND & ND & ND & ND & ND & ND & ND & $\mathrm{ND}$ & ND & ND \\
\hline Bromoform & ND| & $\mathrm{ND}$ & $\mathrm{ND}$ & ND & ND & ND & ND & $\mathrm{ND}$ & ND & $\mathrm{ND}$ & $\mathrm{ND}$ & ND \\
\hline Bromomethane & ND| & $\mathrm{ND}$ & ND & $\mathrm{ND}$ & ND & $\mathrm{ND}$ & ND & ND & ND & ND & ND & ND \\
\hline Carbon disulfide & ND & 0.59 & ND & 1.36 & ND & 2.83 & ND & 1.36 & ND & 1.36 & ND & 1.36 \\
\hline Carbon tetrachloride & $\mathrm{ND}$ & ND & ND & ND & ND & ND| & ND & ND & $\mathrm{ND}$ & ND & ND & 0.27 \\
\hline Chlorobenzene & ND| & ND: & 1.1 & ND & ND & ND| & $\mathrm{ND}$ & ND & $\mathrm{ND}$ & ND & 0.412 & 0.11 \\
\hline Chloroethane & $\mathrm{ND}$ & ND & ND & ND & ND & $\mathrm{ND}$ & ND & ND & ND & ND & ND & ND \\
\hline Chloroethene & ND| & 1.87 & 2.2 & ND & $\mathrm{ND}$ & 2.49 & ND & ND| & 47.2 & $\mathrm{ND}$ & 2.29 & 0.15 \\
\hline Chloroform & $\mathrm{ND}$ & ND & ND & 0.40 & ND & ND & ND & ND & ND & 0.22 & ND & 0.27 \\
\hline Chloromethane & ND & ND & ND & ND & $\mathrm{ND}$ & $\mathrm{ND}$ & ND & ND & $\mathrm{ND}$ & ND & $\mathrm{ND}$ & ND \\
\hline cis-1,3-Dichloropropene & ND & ND & ND & ND & ND & ND & ND & ND & ND & ND & ND| & ND \\
\hline Dibromochloromethane & ND| & ND: & ND & ND & ND & $\mathrm{ND}$ & ND & ND & ND & ND & $\mathrm{ND}$ & ND \\
\hline Dichloromethane & ND| & 3.60 & ND & 1.19 & ND & 14.80 & ND & 1.34 & ND & 1.26 & $\mathrm{ND}$ & 2.64 \\
\hline Ethylbenzene & ND| & ND & $N D$ & 0.16 & ND & ND & ND & ND & 0.202 & $\mathrm{ND}$ & NO & 1.63 \\
\hline Methyl ethyl ketone & ND & 0.92 & ND & 2.48 & ND & 2.80 & ND & ND & $\mathrm{ND}$ & 1.54 & ND & 7.94 \\
\hline Methyl isobutyl ketone & ND & ND & ND & ND & ND & No & ND & $\mathrm{ND}$ & ND| & ND & ND| & ND \\
\hline Styrene & ND & ND & ND & ND & $\mathrm{ND}$ & ND & ND & ND & ND & ND & ND & ND \\
\hline Tetrachloroethylene & 0.495 & 13.70 & 2.59 & ND & 3.02 & 16.40 & ND & 5.07 & 7.34 & ND & 9.12 & 1.35 \\
\hline Toluene & $\mathrm{ND}$ & 0.70 & ND & 1.22 & ND & 0.07 & ND & 0.24 & 0.208 & 0.24 & ND & 4.41 \\
\hline trans-1,3-Dichloropropene & ND & ND & ND & ND & $\mathrm{ND}$ & ND & $\mathrm{ND}$ & ND & ND & ND & ND & ND: \\
\hline Trichloroethylene & 0.762 & 87.50 & 21.6 & $\mathrm{ND}$ & 3.96 & 118.00 & ND) & 0.74 & 39.1 & ND & 8.14 & 3.61 \\
\hline Vinyl acetate & ND & ND & ND & ND & ND & ND & ND & ND & ND & $\mathrm{ND}$ & ND & ND \\
\hline Xylenes & ND & 0.55 & ND & 0.49 & ND & 0.63 & ND & 0.48 & 0.614 & 0.49 & $\mathrm{ND}$ & 16 \\
\hline Melas; ugh & OSनIRA & Pro-lRA & POEERRA| & Pि्थास्स & POनIIRA| & ल्जास् & क्स & $\mathrm{rm}$ & लन & natiat & OGARA & 6 \\
\hline Aluminum & 118 & 774 & 15.8 & 107 & 66.8 & 138 & NDI & 180 & 6930 & 2170 & NDI & 179 \\
\hline Antimony & ND & 1.64] & ND & ND & ND & ND & ND & ND & ND & ND & ND & ND \\
\hline Arsenic & ND & 2.11 & 4.2 & 2.03 & ND & ND & ND & ND & ND & 1.99 & ND & ND \\
\hline Barium & 7.48 & 16.2 & 29 & 31 & 56.9 & 52.2 & 10.6 & 23.2 & 107 & 33.5 & 31.4 & 20.7 \\
\hline Beryllium & ND & ND & $\mathrm{ND}$ & 0.026 & ND & ND & $\mathrm{ND}$ & 0.015 & 0.64 & ND & ND & 0.211 \\
\hline Cadmium & $\mathrm{ND}$ & 0.099 & ND & 0.109 & $\mathrm{ND}$ & 0.23 & ND & 0.319 & $\mathrm{ND}$ & ND & ND & ND \\
\hline Chromium & ND & 2.53 & 1.3 & 2.14 & ND & 2.63 & ND & 1.98 & ND & 7.73 & $\mathrm{ND}$ & 2.67 \\
\hline Cobalt & $\mathrm{ND}$ & 0.239 & ND & 0.672 & ND & 0.558 & ND & 0.523 & 7.15 & 0.324 & $\mathrm{ND}$ & 0.932 \\
\hline Copper & 84.3 & 96.2 & 6.83 & 2.74 & 2.76 & 2.27 & 6.18 & 3.33 & 6.19 & 17.4 & 14.5 & 12.3 \\
\hline Iron & 90.7 & 955 & 339 & 542 & 219 & 39.1 & 117 & 110 & 23300 & 654] & ND & 130 \\
\hline Lead & ND & 5.06 & ND & 2.26 & ND & 3.77 & ND & ND & ND & 16.3 & ND & ND \\
\hline Magnesium & ND & 11400 & ND & 2070 & ND & 4520 & $\mathrm{ND}$ & 905 & ND & 2350 & $\mathrm{ND}$ & 929 \\
\hline Manganese & 2.58 & 8.92 & 13.1 & 17.6 & 172 & 177 & 2.67 & 9.68 & 669 & 14.3 & 107 & 70.1 \\
\hline Mercury & ND & 0.049 & 0.216 & 0.436 & ND & 0.224 & $\mathrm{ND}$ & 0.373 & $\mathrm{ND}$ & 0.215 & ND & 0.112 \\
\hline Nickel & ND & 1.93 & 2.9 & 2.51 & ND & 1.2 & $\mathrm{ND}$ & 1.17 & 28.3 & 5.14 & ND & 2.43 \\
\hline Selenium & ND & ND & ND & ND & ND & ND & ND & 1.45 & $\mathrm{ND}$ & ND & ND & ND \\
\hline Silver & ND & ND| & ND| & ND & ND & ND & ND & ND & ND & ND & $\mathrm{ND}$ & ND \\
\hline Thallium & ND| & ND & ND & ND & ND & ND & $\mathrm{ND}$ & 2.48 & ND & ND & $\mathrm{ND}$ & ND \\
\hline Vanadium & ND & 4.16 & ND & 0.816 & ND & 0.387 & ND & 0.818 & ND & 1.18 & ND & 1.87 \\
\hline Zinc & 33.5 & 14.50 & 27.8 & 67.30 & 22.5 & 9.27 & ND & 7.22 & 107 & 17.3 & .12 .4 & 20.9 \\
\hline Indicator Parameters, ugL & POSAIRA & PF्ञाR & POSFIRA & PTEनाR & POE IRA & Pro-IRA & POSARRA & PTE-IRA & POEARA & म्ब-ारA & POSA-IRA & DOFA \\
\hline Chemical oxygen demand & 10000 & 57300 & 37400 & 27200 & 13000 & 29700 & ND & 12600 & 28000 & 18600 & 10000 & 324000 \\
\hline Chloride & 1320 & 1440 & 4108 & 6050 & 2830 & 2670 & 672 & 3820 & 5240 & 4340 & 6600 & 5770 \\
\hline Nitrate as nitrogen & ND| & 800 & ND & 1210 & ND & 530 & $\mathrm{ND}$ & 1730 & ND & 1180 & ND & 1350 \\
\hline Nitrate-nitrite as nitrogen & 726 & 1110 & 805 & 719 & 349 & 150 & $\mathrm{ND}$ & 39.40 & $\mathrm{ND}$ & 664 & 946 & 1140 \\
\hline pH (pH units) & 6.7 & 6.71 & 5.2 & 5.31 & 5.1 & 5.02 & 5.2 & 7.01 & 4.4 & 5.72 & 5.5 & 5.73 \\
\hline Specific conductance (USCM) & 78 & 142 & 49 & 70.40 & 80 & 94.60 & $\mathrm{ND}$ & 56.40 & 419 & 59.30 & 78 & 70.30 \\
\hline Sulfale & 7200 & 14400 & 7970 & 11700 & 25100 & 28000 & 5850 & 8150 & 188000 & 9850 & 1230 & 9390 \\
\hline Total organic carbon & ND & 18800 & 10000 & 4150 & 5000 & 7980 & ND & 1660 & 16000 & 7590 & 9000 & 2630 \\
\hline Total organic halogen & & 54.90 & 44.3 & 86.10 & 19.7 & 76.60 & 9.74 & 56.90 & 210 & 96.80 & 38.4 & 21.60 \\
\hline
\end{tabular}

ND=Analyzed but not detected.

Green tont indicates decreasing concentration.

Red font indicates increasing concentration. 